PACS 61.41.+e, 64.60.Ak, 64.60.Fr, 11.10.Gh-

\title{
STATISTICS OF MULTICOMPONENT POLYMER STARS
}

\author{
C. vON Ferber ${ }^{(1,2)}$, Yu. Holovatch ${ }^{(3)}$ \\ (1) Fachbereich Physik, Universität Essen, \\ D-45117 Essen, Germany; \\ ${ }^{(2)}$ School of Physics and Astronomy, Tel Aviv University, \\ IL-69978 Tel-Aviv, Israel \\ (3) Institute for Condensed Matter Physics \\ of the Ukrainian Academy of Sciences \\ 1 Svientsitskii St., UA-290011 Lviv, Ukraine
}

Received January 23, 1997

\begin{abstract}
We analyze a polymer network made of chemically different polymer species. Considering the star-like vertices constituting it in order to describe their scaling properties we introduce a new set of critical exponents. In the case of network made of two species of polymers we call them copolymer star exponents. By means of mapping our theory on appropriate Lagrangean field theory we calculate these exponents as well as the exponents governing scaling properties of star of mutually avoiding walks in the third order of perturbation theory. In the case of homogeneous stars we recover previously obtained values of star exponents. Our results agree as well with the previous studies of special cases which were done to the second order of the $\varepsilon$-expansion. We found consistency and stability of the results in $d=2$ and $d=3$ with expected growing of deviations for large number of arms of one star. The same methods were applied previously to the problem of uniform star polymers and have led to results in good agreement with Monte Carlo simulations. We hope our present calculations might also stimulate Monte Carlo studies of the copolymer star problem. The resummed values of the exponents for stars of mutually avoiding walks are in fair agreement with an exact result previously conjectured at $d=2$. The study performed for $d=2$ could give some insight to the problem of mapping our theory to a two dimensional conformal field theory. By studying the convexity properties of the spectrum of copolymer star exponents we show that they are a good candidate for finding application in the theory of multifractal spectra generated by the harmonic diffusion near the absorbing fractal.
\end{abstract}

\section{Introduction}

Polymers and polymer solutions are among the most intensively studied and interesting objects in condensed matter physics. The behaviour of multicomponent solutions containing polymers of different species is especially rich [1]. Of special experimental and technical interest are systems of polymer chains of different species which are linked chemically together. These systems may be block copolymers of two chains of different species linked

(C) C.von Ferber, Yu.Holovatch, 1997

ISSN 0452-9910. Condensed Matter Physics 1997 No 10 (9-40) 
at their endpoints or more generally any number of chains may be linked in the form of stars or networks of any topology (see Figs.1 - 3).

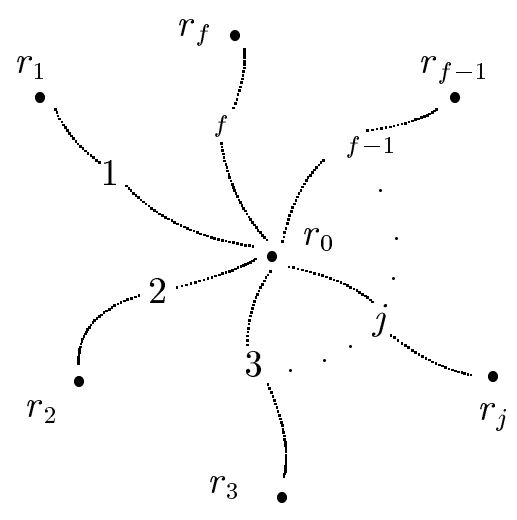

Figure 1. Star polymer of $f$ arms linked together at point $r_{0}$ with extremities placed at points $r_{1} \ldots r_{f}$.

For homogeneous systems of one species the scaling properties of such polymer networks have been extensively studied (for a review see [2]). Star polymers as the most simple polymer networks may be produced by linking together the endpoints of polymer chains at some core molecule (Fig. 1).

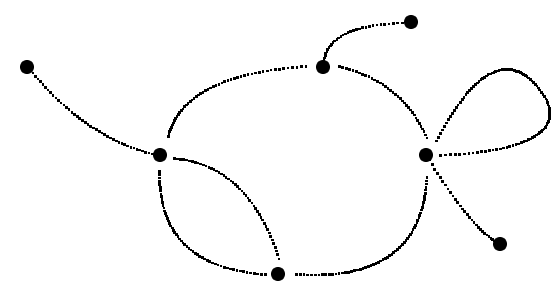

Figure 2. A polymer network $G$. It is characterized by the numbers $n_{f}$ of $f$-leg vertices. Here $n_{1}=3, n_{3}=2, n_{4}=1, n_{5}=1$.

In the same way more general networks of a given topology are formed (Fig. 2). Randomly linked polymer networks on the other hand are obtained as result of a vulcanization process randomly linking nearby monomers of different chains to each other.

The asymptotic properties of homogeneous systems of linear chain molecules in solution are universal in the limit of long chains. For each system there exists a so called $\Theta$ temperature at which the two point attractive and repulsive interactions between the different monomers compensate each other and as a result the polymer chains may be described by random walks (up to higher order corrections): The mean square distance between the chains endpoints $\left\langle R^{2}\right\rangle$ scales with the number of monomers $N$ like $\left\langle R^{2}\right\rangle \sim N$. 
Above the $\Theta$ temperature the effective interaction between the monomers is repulsive resulting in a swelling of the polymer coil which is universal in the asymptotics:

$$
\left\langle R^{2}\right\rangle \sim N^{2 \nu} \text { for } N \rightarrow \infty \text { with } \nu(d=3) \approx 0.588,
$$

$d$ being the dimension of space. The number of configurations $Z$ of a polymer chain of $N$ monomers scales with $N$ like

$$
Z \sim e^{\mu N} N^{\gamma-1}
$$

with a non - universal fugacity $e^{\mu}$. In the early 70-ies following the work of de Gennes [3] the analogy between the asymptotic properties of long polymer chains and the long distance correlations of a magnetic system in the vicinity of the 2nd order phase transition was recognized and elaborated in detail (see $[1,4]$. This mapping allows us to receive the above defined exponents $\nu$ and $\gamma$ as $m \rightarrow 0$ limits of the correlation length exponent $\nu$ and the magnetic susceptibility critical exponent $\gamma$ of the $O(m)$ - symmetric model.

On the other hand, if polymers of different species are present in the same solution the scaling behavior of the observables may be much more rich. Let us consider a solution of two different species of polymers in some solvent, a so called ternary solution. Depending on the temperature the system may then behave as if one or more of the inter- and intra- chain interactions vanish in the sense described above [5-9]. This will lead to asymptotic scaling laws that may differ from those observed for each species alone [10].

Very interesting new systems are obtained when linking together polymers of different species. The most simple system of this kind is a so called block copolymer consisting of two parts of different species. They are of some technical importance e.g. serving as surfactants [11]. For our study they give the most simple example of a polymer star consisting of chains of two different species (Fig. 3a) which we will call here a copolymer star. For the homogeneous polymer star the asymptotic properties are uniquely defined by the number of its constituting chains and the dimension of space $[2,12]$. For the number of configurations $\mathcal{Z}_{f}$ of a polymer star of $f$ chains each consisting of $N$ monomers one finds:

$$
\mathcal{Z}_{f} \sim e^{\mu N f} N^{\gamma_{f}-1}, N \rightarrow \infty .
$$

The exponents $\gamma_{f}, f=1,2,3, \ldots$ constitute a family of star exponents, which depend on the number of arms $f$ in a nontrivial way. The case of linear polymer chains is included in this family with the exponent $\gamma=\gamma_{1}=\gamma_{2}$ defined in (1.2). For general numbers of arms $f$ the star exponents $\gamma_{f}$ have no physical counterparts in the set of exponents describing magnetic phase transitions. Nevertheless they can be related to the scaling dimensions of composite operators of traceless symmetry in the polymer limit $m \rightarrow 0$ of the $O(m)$ symmetric $m$ vector model [13,14]. These exponents have been calculated analytically in perturbation theory $[12,13,15,16]$, by exact methods in two dimensions [12,17], and by Monte Carlo simulations [18-20].

It has been shown that the scaling properties of polymer networks of arbitrary but fixed topology are uniquely defined by its constituting stars [2], as long as the statistical ensemble respects some conditions on chain length distribution [13]. Thus the knowledge of the set of star exponents allows to obtain the power laws corresonding to (1.3) also for any polymer network of arbitrary topology. 


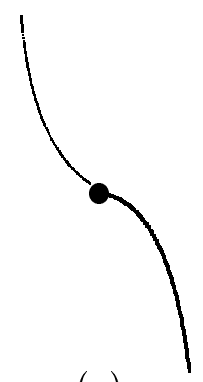

(a)

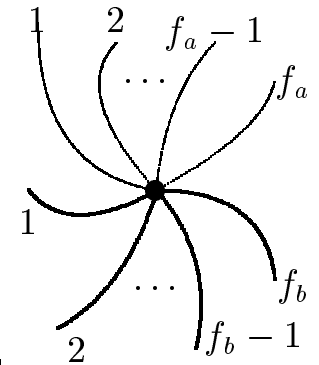

(b)

Figure 3. a. Block copolymer consisting of two polymer chains of different species (shown by solid and dashed curves) linked at their endpoints. $b$. Copolymer star consisting of $f_{a}$ arms of species $a$ and $f_{b}$ arms of species $b$ tied together at their endpoints.

In this article we address a somewhat more complex problem: What happens to the scaling laws if we build a polymer star or general network of chains of different species? In view of the above introduced ternary solutions, one may thus study sytems of polymer networks in which some of the intra and inter chain interactions vanish. For example one may descibe a copolymer star in solution consisting of say $f_{a}$ chains of species $a$ and $f_{b}$ chains of species $b$ (see Fig. 3b) in the situation that there are no interactions among the chains of each species alone but only between chains of different species $a$ and $b$. This situation in turn may also be interpreted as a number $f_{b}$ of random walks which end at the core of a star of $f_{a}$ chains with the constraint that the $f_{b}$ random walks avoid the chains of the star. One may thus describe diffusion phenomena with some complicated boundary condition and find a tractable case of the more complex growth phenomena in a Laplacian field $[21,22]$.

The setup of our article is as follows. In section 2. we introduce notation and relate the polymer model to a Lagrangean field theory. This field theoretical formalism will be used throughout the paper. In section 3. we define the renormalization group procedures. We present two alternative approaches: zero mass renormalization together with $\varepsilon$ - expansion see (e.g. [23]) and massive renormalization at fixed dimension [24]. Section 4. is devoted to the study of the renormalization group flow of the ternary model and its fixed points. Series for critical exponents governing the scaling behavior of copolymer stars and stars of mutually avoiding walks are obtained in section 5.. In section 6 . we discuss the problem of resummation of the asymptotic series arising in this context. Numerical results are presented in section 7 . We close with concluding remarks and an outlook on possible applications of the theory in section 8. and give some calculational details in appendices. Some of our principal results have previously been announced in a Letter [25].

\section{Model and Notations}

Let us first take a look at the model we use to describe polymers. In a first discrete version we will describe a configuration of the polymer by a set of 
positions of segment endpoints:

$$
\text { Configuration }\left\{r_{1}, \ldots, r_{N}\right\} \in \mathbb{R}^{d \times N} .
$$

Its statistical weight (Boltzmann factor) with the Hamiltonian $\mathcal{H}$ divided by the product of Boltzmann constant $k_{\mathrm{B}}$ and temperature $T$ will be given by

$$
\exp \left[-\frac{1}{k_{\mathrm{B}} T} \mathcal{H}\right]=\exp \left\{-\frac{1}{4 \ell_{0}^{2}} \sum_{j=1}^{N}\left(r_{j}-r_{j-1}\right)^{2}-\beta \ell_{0}^{d} \sum_{i \neq j=1}^{N} \delta^{d}\left(r_{i}-r_{j}\right)\right\} .
$$

The first term describes the chain connectivity, the parameter $\ell_{0}$ governing the mean segment length. The second term describes the excluded volume interaction forbidding two segment end points to take the same position in space. The parameter $\beta$ gives the strength of this interaction. The third parameter in our model is the chain length or number of segments $N$.

The partition sum $\mathcal{Z}$ will be calculated as an integral over all configurations of the polymer divided by the system volume $\Omega$, thus dividing out identical configurations just translated in space [26]:

$$
\mathcal{Z}(N)=\frac{1}{\Omega} \int \prod_{i=1}^{N} \mathrm{~d} r_{i} \exp \left[-\frac{1}{k_{\mathrm{B}} T} \mathcal{H}\left\{r_{i}\right\}\right]
$$

This will give us the 'number of configurations' of the polymer (1.2). We will do our investigations by mapping the polymer model to a renormalizable field theory making use of well developed formalisms (see [1,4] for example). To this end we introduce a continuous version of our model as proposed by Edwards $[27,28]$ generalizing it to describe a set of $f$ polymer chains of varying composition possibly tied together at their end points. The configuration of one polymer is now given by a path $r^{a}(s)$ in $d$ - dimensional space $\mathbb{R}^{d}$ parametrized by a surface variable $0 \leq s \leq S_{a}$. The relation of the 'Gaussian surface' $S_{a}$ of the chain $a$ to the number of segments $N_{a}$ in the discrete model is $S_{a}=N \ell_{0}^{2}$. We now allow for a symmetric matrix of excluded volume interactions $u_{a b}$ between chains $a, b=1, \ldots, f$. The Hamiltonian $\mathcal{H}$ is then given by

$$
\frac{1}{k_{\mathrm{B}} T} \mathcal{H}\left(r^{a}\right)=\sum_{a=1}^{f} \int_{0}^{S_{a}} \mathrm{~d} s\left(\frac{\mathrm{d} r^{a}(s)}{\mathrm{d} s}\right)^{2}+\frac{1}{6} \sum_{a, b=1}^{f} u_{a b} \int \mathrm{d}^{d} r \rho_{a}(r) \rho_{b}(r),
$$

with densities $\rho_{a}(r)=\int_{0}^{S_{a}} \mathrm{~d} s \delta^{d}\left(r-r^{a}(s)\right)$. Now the partition sum has to be calculated using a functional integral:

$$
\mathcal{Z}_{f}\left\{S_{a}\right\}=\frac{1}{\Omega} \int \mathcal{D}\left[r^{a}(s)\right] \exp \left\{-\frac{1}{k_{\mathrm{B}} T} \mathcal{H}\left(r^{a}\right)\right\} .
$$

here the symbol $\mathcal{D}\left[r_{a}(s)\right]$ includes normalization such that $Z\left\{S_{a}\right\}=1$ for all $u_{a b}=0$. To make the exponential of $\delta$-functions in (2.4) and the functional integral well-defined in the bare theory a cutoff $s_{0}$ is to be introduced such that all simultaneous integrals of any variables $s$ and $s^{\prime}$ are cut off by | $s-s^{\prime} \mid>s_{0}$. Let us note here that equation (2.4) describing a system of continuous chains may be understood as a limit of discrete self-avoiding 
walks, when the length of each step is decreasing while the number of steps $N_{a}$ is increasing keeping the mean square size of chain fixed. Thus one can relate the Gaussian surface $S_{a}$ of each path to the notion of steps by

$$
N_{a}=S_{a} / s_{0}
$$

Now the continuous chain model (2.3) can be mapped onto a corresponding field theory by a Laplace transform in the Gaussian surfaces $S_{a}$ to conjugate chemical potentials ("mass variables") $\mu_{a}$ :

$$
\tilde{\mathcal{Z}}_{f}\left\{\mu_{a}\right\}=\int_{0}^{\infty} \prod_{b} \mathbf{d} S_{b} e^{-\mu_{b} S_{b}} \mathcal{Z}_{f}\left\{S_{a}\right\}
$$

where the Laplace-transformed partition function $\tilde{\mathcal{Z}}_{f}\left\{\mu_{a}\right\}$ can be expressed as the $m=0$ limit of the functional integral over vector fields $\phi_{a}, a=$ $1, \ldots, f$ with $m$ components $\phi_{a}^{\alpha}, \alpha=1, \ldots, m$ :

$$
\tilde{\mathcal{Z}}_{f}\left\{\mu_{b}\right\}=\left.\frac{1}{\Omega} \int \mathcal{D}\left[\phi_{a}(r)\right] \exp \left[-\mathcal{L}\left\{\phi_{b}, \mu_{b}\right\}\right]\right|_{m=0} .
$$

the Landau-Ginzburg-Wilson-Lagrangean $\mathcal{L}$ of $f$ interacting fields $\phi_{b}$ each with $m$ components reads

$$
\mathcal{L}\left\{\phi_{b}, \mu_{b}\right\}=\frac{1}{2} \sum_{a=1}^{f} \int \mathrm{d}^{d} r\left(\mu_{a} \phi_{a}^{2}+\left(\nabla \phi_{a}(r)\right)^{2}\right)+\frac{1}{4 !} \sum_{a, a^{\prime}=1}^{f} u_{a, a^{\prime}} \int \mathrm{d}^{d} r \phi_{a}^{2}(r) \phi_{a^{\prime}}^{2}(r) .
$$

here

$$
\phi_{a}^{2}=\sum_{\alpha=1}^{m}\left(\phi_{a}^{\alpha}\right)^{2} .
$$

The limit $m=0$ in (2.6) can be understood as a certain rule to calculate the diagrams appearing in the perturbation theory expansions and can be easily checked diagrammatically. A rigorous proof based on the application of Stratonovich-Hubbard transformation to linearize terms in (2.3) is given for the multicomponent case in [10].

The one particle irreducible vertex function $\Gamma^{(L)}\left(q_{i}\right)$ can be defined by:

$$
\delta\left(\sum q_{i}\right) \Gamma^{(L)}\left(q_{i}\right)=\int e^{i q_{i} r_{i}} \mathrm{~d} r_{1} \ldots \mathrm{d} r_{L}\left\langle\phi_{a_{1}}\left(r_{1}\right) \ldots \phi_{a_{L}}\left(r_{L}\right)\right\rangle_{1 P I}^{\mathcal{L}} .
$$

Averaging in (2.9) is done with respect to the Lagrangean (2.7) while keeping only those contributions which correspond to one-particle irreducible graphs.

The partition function $Z_{* f}\left\{S_{a}\right\}$ of a polymer star consisting of $f$ polymers of different species $1, \ldots, f$ constrained to have a common end point is obtained from (2.4) by introducing an appropriate product of $\delta$-functions ensuring the "star-like" structure. It reads:

$$
\begin{aligned}
& Z_{* f}\left\{S_{a}\right\}=\frac{1}{\Omega} \int \mathcal{D}\left[r_{a}\right] \exp \left\{-\sum_{a=1}^{f} \int_{0}^{S_{a}} \mathrm{~d} s\left(\frac{\mathrm{d} \vec{r}_{a}(s)}{\mathrm{d} s}\right)^{2}-\right. \\
& \left.\frac{1}{6} \sum_{a, b=1}^{f} u_{a b}^{0} \int \mathrm{d}^{d} r \rho_{a}(\vec{r}) \rho_{b}(\vec{r})\right\} \prod_{a=2}^{f} \delta^{d}\left(\vec{r}_{a}(0)-\vec{r}_{1}(0)\right) .
\end{aligned}
$$


The vertex part of its Laplace transformation may be defined by:

$$
\begin{aligned}
& \delta\left(p+\sum q_{i}\right) \Gamma_{a_{1} \ldots a_{f}}^{(* f)}\left(p, q_{1} \ldots q_{f}\right)=\int e^{i\left(p r_{0}+q_{i} r_{i}\right)} \mathrm{d}^{d} r_{0} \mathrm{~d}^{d} r_{1} \ldots \mathrm{d}^{d} r_{f} \\
& \left\langle\phi_{a_{1}}\left(r_{0}\right) \ldots \phi_{a_{f}}\left(r_{0}\right) \phi_{a_{1}}\left(r_{1}\right) \ldots \phi_{a_{f}}\left(r_{f}\right)\right\rangle_{1 P I}^{\mathcal{L}},
\end{aligned}
$$

where all $a_{1}, \ldots a_{f}$ are distinct. When only species $a$ is present one can also define $\Gamma^{* f}$ as the $m=0$ component limit of

$$
\begin{aligned}
& \delta\left(p+\sum q_{i}\right) \Gamma^{(* f)}\left(q, p_{1} \ldots p_{f}\right)=\int e^{i\left(q r_{0}+p_{i} r_{i}\right)} \mathrm{d}^{d} r_{0} \mathrm{~d}^{d} r_{1} \ldots \mathrm{d}^{d} r_{f} \\
& \left\langle N^{\alpha_{1} \ldots \alpha_{f}} \phi_{a \alpha_{1}}\left(r_{0}\right) \ldots \phi_{a \alpha_{f}}\left(r_{0}\right) \phi_{a \alpha_{1}}\left(r_{1}\right) \ldots \phi_{a \alpha_{f}}\left(r_{f}\right)\right\rangle_{1 P I}^{\mathcal{L}},
\end{aligned}
$$

where $\alpha$ is index of the field component and tensor $N^{\alpha_{1}, \ldots \alpha_{f}}$ has traceless symmetry [14]:

$$
\sum_{\alpha} N^{\alpha \alpha \alpha_{3} \ldots \alpha_{f}}=0 .
$$

In what follows below we will be mainly interested in the case when only two species of polymers are present, with interactions $u_{11}, u_{22}$ between polymers of the same species and $u_{12}=u_{21}$ between polymers of different species. Thus we will present here results for the case of a ternary polymer solution. Nevertheless the results obtained are easily generalized to the case of higher numbers of polymer species.

Let us give the expressions to the third order for those vertex functions we are going to deal with in our study. They involve the loop integrals $D_{2}, I_{1}-I_{8}$ which are given in the Appendix A together with their correspondence to the graphs of perturbation theory. The bare Gamma functions read:

$$
\begin{aligned}
& \quad \frac{\partial}{\partial k^{2}} \Gamma_{(a a)}^{(2)}=1-\frac{1}{9} I_{2} u_{a a}^{2}+\frac{4}{27} I_{8} u_{a a}^{3}, \quad a=1,2 \\
& \Gamma_{(a a a a)}^{(4)}=u_{a a}-\frac{4}{3} D_{2}^{a a} u_{a a}^{2}+\left(\frac{5}{9} D_{2}^{2}+\frac{22}{9} I_{1}\right) u_{a a}^{3}-\left(\frac{2}{9} D_{2}^{3}+\frac{28}{27} I_{1} D_{2}+\right. \\
& \left.\quad \frac{8}{27} I_{3}+\frac{40}{9} I_{4}+\frac{58}{27} I_{5}+\frac{14}{27} I_{6}+\frac{22}{27} I_{7}\right) u_{a a}^{4}, \quad a=1,2, \quad(2.15) \\
& \quad+\left(I_{1}+D_{2}^{2}\right) \bar{u}_{a_{1} a_{2}}^{2} / 2+\bar{u}_{a_{1} a_{1}} \bar{u}_{a_{1} a_{2}} I_{1}+D_{2}^{3} \bar{u}_{a_{1} a_{2}} \bar{u}_{a_{3} a_{4} a_{3}} \bar{u}_{a_{5} a_{6}} / 48 \\
& \quad+D_{2} \bar{u}_{a_{1} a_{2}} \bar{u}_{a_{1} a_{3}} \bar{u}_{a_{4} a_{5}} I_{1} / 2+\bar{u}_{a_{1} a_{2}} \bar{u}_{a_{1} a_{3}} \bar{u}_{a_{3} a_{4}} I_{4} \\
& \quad+\left(I_{5}+I_{6}\right) \bar{u}_{a_{1} a_{2}} \bar{u}_{a_{1} a_{3}} \bar{u}_{a_{2} a_{4}} / 2+\bar{u}_{a_{1} a_{2}} \bar{u}_{a_{1} a_{3}} \bar{u}_{a_{1} a_{4}} I_{4} \\
& \quad+\left(3 I_{4}+I_{7}\right) \bar{u}_{a_{1} a_{2}} \bar{u}_{a_{1} a_{3}} \bar{u}_{a_{2} a_{3}} / 3+D_{2}\left(I_{1}+D_{2}^{2}\right) \bar{u}_{a_{1} a_{2}}^{2} \bar{u}_{a_{3} a_{4}} / 4 \\
& \quad+\left(D_{2} I_{1}+2 I_{4}+2 I_{5}+I_{7}\right) \bar{u}_{a_{1} a_{2}}^{2} \bar{u}_{a_{1} a_{3}}+\left(I_{3}+3 I_{4}+I_{5}\right) \bar{u}_{a_{1} a_{1}}^{2} \bar{u}_{a_{1} a_{2}} \\
& \quad+\left(D_{2} I_{1}+2 I_{4}+I_{5}+I_{6}+D_{2}^{3}\right) \bar{u}_{a_{1} a_{2}}^{3} / 2+D_{2} \bar{u}_{a_{1} a_{1}} \bar{u}_{a_{1} a_{2}} \bar{u}_{a_{3} a_{4}} I_{1} / 2 \\
& \quad+\left(I_{4}+I_{5}\right) \bar{u}_{a_{1} a_{1}} \bar{u}_{a_{1} a_{2}} \bar{u}_{a_{2} a_{3}}+\left(I_{4}+I_{5}+I_{7}\right) \bar{u}_{a_{1} a_{1}} \bar{u}_{a_{1} a_{2}} \bar{u}_{a_{1} a_{3}} \\
& \quad+\bar{u}_{a_{1} a_{1}} \bar{u}_{a_{1} a_{2}} \bar{u}_{a_{2} a_{2}} I_{5} / 2+\left(D_{2} I_{1}+4 I_{4}+I_{6}\right) \bar{u}_{a_{1} a_{1}} \bar{u}_{a_{1} a_{2}}^{2}
\end{aligned}
$$


Here summation over $a_{i}=1 \ldots f$ is assumed. For a star of $f_{1}$ chains of species 1 and $f_{2}$ chains of species 2 the matrix of interactions $\bar{u}_{a b}$ is given by

$$
\bar{u}_{a b}^{f_{1} f_{2}}= \begin{cases}u_{11} & 1 \leq a, b \leq f_{1} \\ u_{22} & f_{1}<a, b \leq f \\ u_{12} & \text { else }\end{cases}
$$

Let us define in this way:

$$
\Gamma^{\left(* f_{1} f_{2}\right)}=\left.\Gamma^{(* f)}\right|_{\bar{u}_{a b}=\bar{u}_{a b}^{f_{1} f_{2}}}
$$

For general $f_{1}, f_{2}$ the corresponding combinatorics may also be directly calculated by summation over $a_{i}=1,2$ instead. Replacing $v=u$, each term in the sum with indices $a_{1} \ldots a_{k}$ then aquires a factor

$$
\left(\begin{array}{c}
f_{1} \\
\#_{1}\left(a_{1} \ldots a_{k}\right)
\end{array}\right)\left(\begin{array}{c}
f_{2} \\
\#_{2}\left(a_{1} \ldots a_{k}\right)
\end{array}\right) \text {. }
$$

Here $\#_{1}\left(a_{1} \ldots a_{k}\right)$ is the number of $a_{i}=1$ whereas $\#_{2}\left(a_{1} \ldots a_{k}\right)$ is the number of $a_{i}=2$.

As a special case we may derive the vertex function $\Gamma_{1122}^{(4)}$ for the $u_{12}$ interaction using the relation $\Gamma^{(* 22)}=\partial / \partial u_{12} \Gamma_{1122}^{(4)}$ which is obvious from the perturbation theory (see [31] for instance):

$$
\Gamma_{1122}^{(4)}=\int \mathrm{d} u_{12} \Gamma^{(* 22)}
$$

With the same formalism we can also describe a star of $f$ mutually avoiding walks [29]. In this case all interactions on the same chain $v_{a a}$ vanish and only those $\bar{u}_{a b}$ with $a \neq b$ remain:

$$
\Gamma_{\mathrm{MAW}}^{(* f)}=\left.\Gamma^{(* f)}\right|_{\bar{u}_{a b}=\left(1-\delta_{a b}\right) u_{12}} .
$$

In this case each term with indices $a_{1} \ldots a_{k}$ aquires a factor $\left(\begin{array}{l}f \\ k\end{array}\right) k$ !.

As it is well known ultraviolet divergencies occur when the vertex functions (2.14) - (2.16) are evaluated naively [30]. In the next section the field theoretical renormalization group approach is to be applied to study this problem.

\section{Renormalization}

We apply renormalization group (RG) theory to make use of the scaling symmetry of the systems in the asymptotic limit to extract the universal content and at the same time remove divergencies which occur for the evaluation of the bare functions in this limit $[23,30,31]$. We pass from the theory in terms of the initial bare variables to a renormalized theory. This can be achieved by a controlled rearrangement of the series for the vertex functions. Serveral asymptotically equivalent procedures serve to this purpose. Here we will use two somewhat complementary approaches: zero mass renormalization (see [23] for instance) with successive $\varepsilon$-expansion [32] and the fixed dimension RG approach [24]. The first is defined directly for the critical point but on the other hand has to make use of the $\varepsilon=4-d$ expansion 
in order to give results for critical exponents at physically interesting dimensions $d=2$ and $d=3$ [32-35]. The second approach renormalizes for non zero mass and leads to results for critical exponents directly in space dimensions $d=2, d=3[36,37]$ but can not be performed at the critical point itself though leading to quantitative results for preasymptotic critical behaviour [38,39]. Most authors tend to prefer one method and to exclude the other for non obvious reasons. The application of both approaches will enable us in particular to check the consistency of approximations and the accuracy of the results obtained.

Let us formulate the relations to obtain a renormalized theory in terms of corresponding renormalization conditions. Though being different in principle for the two procedures, we may formulate them simultaneously using the same expressions. Note that the polymer limit of zero component fields leads to essential simplification. Each field $\phi_{a}$, mass $m_{a}$ and coupling $u_{a a}$ renormalizes as if the other fields were absent. First we introduce renormalized couplings $g_{a b}$ by:

$$
\begin{aligned}
& u_{a a}=\mu^{\varepsilon} Z_{\phi_{a}}^{2} Z_{a a} g_{a a}, a=1,2 \\
& u_{12}=\mu^{\varepsilon} Z_{\phi_{1}} Z_{\phi_{2}} Z_{12} g_{12} .
\end{aligned}
$$

Here, $\mu$ is a scale parameter (equal to the mass at which the massive scheme is evaluated and giving the scale of external momenta in the massless scheme). The renormalization factors $Z_{\phi_{a}}, Z_{a b}$ are defined as power series in the renormalized coupling which fulfill the following RG conditions:

$$
\begin{gathered}
Z_{\phi_{a}}\left(g_{a a}\right) \frac{\partial}{\partial k^{2}} \Gamma_{a a}^{(2)}\left(u_{a a}\left(g_{a a}\right)\right)=1 \\
Z_{a a}\left(g_{a a}\right) \Gamma_{a a a a}^{(4)}\left(u_{a a}\left(g_{a a}\right)\right)=\mu^{\varepsilon} g_{a a} \\
Z_{12}\left(g_{a b}\right) \Gamma_{1122}^{(4)}\left(u_{a b}\left(g_{a b}\right)\right)=\mu^{\varepsilon} g_{12}
\end{gathered}
$$

Evaluating these formulas perturbatively the corresponding loop integrals are evaluated in the massive approach for zero external momenta and in the massless approach for external momenta at the scale of $\mu$ as given in the appendix A. In the first case the RG condition for the vertex function $\Gamma^{(2)}$ reads

$$
\left.\Gamma_{a a}^{(2)}\left(u_{a a}\left(g_{a} a\right)\right)\right|_{k^{2}=0}=m_{a}^{2}, a=1,2
$$

in the second case of massles renormalization the corresponding condition reads

$$
\left.\Gamma_{a a}^{(2)}\left(u_{a a}\left(g_{a a}\right)\right)\right|_{k^{2}=\mu^{2}}=0, a=1,2
$$

In order to renormalize the star vertex functions we introduce renormalization factors $Z_{* f_{1}, f_{2}}$ by

$$
Z_{\phi_{1}}^{f_{1} / 2} Z_{\phi_{2}}^{f_{2} / 2} Z_{* f_{1}, f_{2}} \Gamma^{\left(* f_{1} f_{2}\right)}\left(u_{a b}\left(g_{a b}\right)\right)=\mu^{\delta_{\left(f_{1}+f_{2}\right)}} .
$$

In the same way we define the appropriate renormalization of the mutually avoiding walks (MAW) vertex function:

$$
Z_{\phi_{1}}^{f / 2} Z_{(\mathrm{MAW} f)} \Gamma_{\mathrm{MAW}}^{* f}\left(u_{12}\left(g_{a b}\right)\right)=\mu^{\delta_{f}} .
$$


The powers of $\mu$ absorb the engeneering dimensions of the bare vertex functions. These are given by

$$
\delta_{f}=f(\varepsilon / 2-1)+4-\varepsilon .
$$

The renormalized couplings $g_{a b}$ defined by the relations (3.1),(3.2) depend on the scale parameter $\mu$. By their dependence on $g_{a b}$ also the renormalization $Z$ - factors depend implictly on $\mu$. This dependence is expressed by the renormalization group functions defined by the following relations:

$$
\begin{aligned}
\mu \frac{\mathrm{d}}{\mathrm{d} \mu} g_{a b} & =\beta_{a b}\left(g_{a^{\prime} b^{\prime}}\right) \\
\mu \frac{\mathrm{d}}{\mathrm{d} \mu} \ln Z_{\phi_{a}} & =\eta_{\phi_{a}}\left(g_{a a}\right) \\
\mu \frac{\mathrm{d}}{\mathrm{d} \mu} \ln Z_{* f_{1} f_{2}} & =\eta_{* f_{1} f_{2}}\left(g_{a b}\right) \\
\mu \frac{\mathrm{d}}{\mathrm{d} \mu} \ln Z_{\mathrm{MAW} f} & =\eta_{f}^{\mathrm{MAW}}\left(g_{a b}\right)
\end{aligned}
$$

The function $\eta_{\phi_{a}}$ describes the pair correlation critical exponent, while the functions $\eta_{* f_{1} f_{2}}$ and $\eta_{f}^{\mathrm{MAW}}\left(g_{a b}\right)$ define the set of exponents for copolymer stars and stars of mutually avoiding walks. Explicit expressions for the $\beta$ and $\eta$ functions will be given in the next section together with a study of the RG flow and the fixed points of the theory.

\section{Renormalization Group Flow and the Fixed Points: $\varepsilon$ - expansion and pseudo- $\varepsilon$-expansion}

Here we want to discuss the RG flow of the theory presented in section 3.. In particular we want to find appropriate representations for the fixed points of the flow for both approaches used here. In a study devoted to ternary polymer solutions the RG flow has previously been calculated [10] in the frames of massless renormalization and given to third loop order in the $\varepsilon$-expansion. Note that for the diagonal coupling $g_{a a}$ the corresponding expressions are also found in the polymer limit $m=0$ of the $O(m)$-symmetrical $\phi^{4}$ model. They are known in even higher orders of perturbation theory [40]. To third loop order the expressions read:

$$
\begin{aligned}
& \beta_{g_{a a}}^{\varepsilon}=-\varepsilon g_{a a}+\frac{1}{3}\left(4+2 \varepsilon+2 \varepsilon^{2}\right) g_{a a}^{2}-\frac{1}{9}\left(\frac{21}{2}+\frac{215}{8} \varepsilon-11 J \varepsilon\right) g_{a a}^{3}+ \\
& \frac{1}{27}(79-22 J+33 \zeta(3)) g_{a a}^{4}+O\left(g_{a a}^{5}\right), \quad a=1,2 . \\
& \beta_{g_{12}}^{\varepsilon}=-\varepsilon g_{12}+\frac{1}{3}\left(1+\frac{\varepsilon}{2}+\frac{\varepsilon^{2}}{2}\right)\left(g_{11}+g_{22}\right) g_{12}+\frac{1}{3}\left(2+\varepsilon+\varepsilon^{2}\right) g_{12}^{2}- \\
& \frac{1}{9}\left(\frac{5}{4}+\frac{55}{16} \varepsilon-\frac{3}{2} J \varepsilon\right)\left(g_{11}^{2}+g_{22}^{2}\right) g_{12}-\frac{1}{9}\left(3+\frac{15}{2} \varepsilon-3 J \varepsilon\right)\left(g_{11}+g_{22}\right) g_{12}^{2}- \\
& \frac{1}{9}(2+5 \varepsilon-2 J \varepsilon) g_{12}^{3}+\frac{1}{54}(15-J)\left(g_{11}^{3}+g_{22}^{3}\right) g_{12}+\frac{1}{27}\left(\frac{27}{2}+\right. \\
& 9 \zeta(3)-6 J)\left(g_{11}^{2}+g_{22}^{2}\right) g_{12}^{2}+\frac{1}{27}(7-3 J) g_{11} g_{22} g_{12}^{2}+\frac{1}{27}(12+6 \zeta(3)- \\
& 2 J)\left(g_{11}+g_{22}\right) g_{12}^{3}+\frac{1}{27}(6+3 \zeta(3)-2 J) g_{12}^{4}+O\left(g^{5}\right) .
\end{aligned}
$$


Here the Riemann $\zeta$-function with $\zeta(3) \approx 1.202$ and the constant $J \approx 0.7494$ occur. The index $\varepsilon$ at $\beta^{\varepsilon}$ is used to distinguish the $\beta$-functions obtained in massless renormalization with successsive $\varepsilon$-expansion from $\beta^{m}$ obtained in massive field theory.

Similarlily, performing renormalization in the massive scheme, as described in the previous section we obtain the corresponding functions $\beta^{m}$. In order to present them let us pass to new variables $v_{a b}=D_{2}^{m} g_{a b}$ and introduce new functions $\beta_{v_{a b}}^{m}=D_{2}^{m} \beta_{g_{a b}}^{m}, D_{2}^{m}$ is the one-loop integral calculated in massive field theory framework (see appendix A). This defines a convenient numerical scale for $\beta$-function. The expression for functions $\beta_{v_{a b}}^{m}$ read:

$$
\begin{aligned}
& \beta_{v_{a a}}^{m}=-(4-d) v_{a a}\left(1-\frac{4 v_{a a}}{3}+\frac{2}{9}\left(22\left(i_{1}-\frac{1}{2}\right)+2 i_{2}\right) v_{a a}^{2}+\frac{2}{27}(-89+\right. \\
& 310 i_{1}+8 i_{2}+3 i_{2} d-12 i_{3}-180 i_{4}-87 i_{5}-21 i_{6}-33 i_{7}- \\
& \left.\left.12 i_{8}\right) v_{a a}^{3}\right), \quad a=1,2 . \\
& \beta_{v_{12}}^{m}=-(4-d) v_{12}\left(1-\frac{1}{3}\left(v_{11}+v_{22}+2 v_{12}\right)+\frac{1}{3}\left(-v_{11}^{2}-v_{22}^{2}-2 v_{12} v_{11}\right.\right. \\
& -\frac{4 v_{12}^{2}}{3}-2 v_{12} v_{22}+\frac{2 v_{12}^{2} i_{2}}{3}+\frac{8 v_{12}^{2} i_{1}}{3}+4 v_{12} v_{22} i_{1}+2 v_{11}^{2} i_{1}+\frac{2 v_{22}^{2} i_{2}}{3}+ \\
& \left.\left.4 v_{12} v_{11} i_{1}+2 v_{22}^{2} i_{1}\right)+\sum_{j=0}^{8} b_{v_{12}}^{j} i_{j}\right)
\end{aligned}
$$

where $i_{j}$ are the dimension-dependent loop integrals entering the expressions for the bare vertex functions (2.14)-(2.16) normalized by the one-loop integral value (see appendix A). Expressions for the coefficients $b_{v_{12}}^{j}$ are given in the appendix B. Again, in the case of polymer limit of $O(m)$ symmetric $\phi^{4}$ model (formula (4.3)) we recover to the third order expressions for $\beta$-function obtained in the massive scheme in [36] for $d=3$ in six-loop order [41].

The equations for the fixed points $\left\{g_{11}^{*}, g_{22}^{*}, g_{12}^{*}\right\}$ of the functions $\beta^{\varepsilon}$ as obtained in massless renormalization,

$$
\begin{aligned}
\beta_{g_{a a}}^{\varepsilon}\left(g_{a a}^{*}\right) & =0, \quad a=1,2, \\
\beta_{g_{12}}^{\varepsilon}\left(g_{11}^{*}, g_{22}^{*}, g_{12}^{*}\right) & =0
\end{aligned}
$$

have 8 different solutions, corresponding to the different cases of interacting and non-interacting chains. In the case when the interaction between the chains of different species is absent $\left(g_{12}^{*}=0\right)$ one finds the following fixed points $G_{0}\left(g_{11}^{*}=0, g_{22}^{*}=0\right), U_{0}\left(g_{11}^{*} \neq 0, g_{22}^{*}=0\right), U_{0}^{\prime}\left(g_{11}^{*}=0, g_{22}^{*} \neq 0\right)$, $S_{0}\left(g_{11}^{*} \neq 0, g_{22}^{*} \neq 0\right)$.

It is clear from the expressions (4.1) for the $\beta$-functions that non-zero solutions for $g_{11}^{*}, g_{22}^{*}$ in (4.5) correspond to the fixed points describing selfavoiding walks (zero solutions correspond to random walks). More interesting are the fixed points corresponding to the case of mutually interacting chains $\left(\left(g_{12}^{*} \neq 0\right)\right)$. Keeping notations of $[10]$ they are: $G\left(g_{11}^{*}=0, g_{22}^{*}=0\right)$, $U\left(g_{11}^{*} \neq 0, g_{22}^{*}=0\right), U^{\prime}\left(g_{11}^{*}=0, g_{22}^{*} \neq 0\right), S\left(g_{11}^{*} \neq 0, g_{22}^{*} \neq 0\right)$.

In the three dimensional space of couplings $g_{11}, g_{22}, g_{12}$ the above discussed fixed points are placed at the corners of a cube deformed in the $g_{12}$ direction [10] (see figure 4). Their numerical values in $\varepsilon$-expansion are given 
in Table 1, with

$$
\begin{aligned}
& g_{G}^{*}=\frac{3 \varepsilon}{2}-\left(J+\frac{3}{2} \zeta(3)\right) \frac{3 \varepsilon^{3}}{8} \\
& g_{U}^{*}=\frac{9 \varepsilon}{8}+\frac{39 \varepsilon^{2}}{256}+\left(\frac{267}{4096}-\frac{693}{1024} \zeta(3)-\frac{189}{512} J\right) \varepsilon^{3} \\
& g_{S}^{*}=\frac{3 \varepsilon}{4}+\frac{15 \varepsilon^{2}}{128}+\left(\frac{111}{2048}-\frac{99}{256} \zeta(3)-\frac{33}{128} J\right) \varepsilon^{3}
\end{aligned}
$$

Being interested in numerical values of the fixed points of $\beta$-functions obtained in the massive scheme (4.3), (4.4) (as well as in the other quantities of the theory) one has several alternatives in analysing the equations (4.3), (4.4). The first possibility is to introduce the $\varepsilon$-expansions for the loop integrals. For massive renormalization these are known for the one- and two-loop integrals (see [31]):

$$
D_{2}^{m}=\frac{1}{\varepsilon}\left(1-\frac{\varepsilon}{2}\right)+O(\varepsilon), \quad i_{1}=\frac{1}{2}+\frac{\varepsilon}{4}+O\left(\varepsilon^{2}\right), \quad i_{2}=-\frac{\varepsilon}{8}+O\left(\varepsilon^{2}\right),
$$

A second possibility is to proceed directly in fixed dimension of space $d=2$ or $d=3$ substituting instead of the loop integrals their numerical values in fixed dimensions $[42,43]$. For the first case ( $\varepsilon$-expansion) the nontrivial (i.e. $v_{12}^{*} \neq 0$ ) fixed points are given in table 2 to 2 nd order in $\varepsilon$. Comparing these results to the corresponding fixed point values obtained in [10] in the massless renormalization scheme (Table 1 and formulas (4.6)(4.8) ) one can see that only the terms linear in $\varepsilon$ coincide and a difference arises already in the $\varepsilon^{2}$ terms. It is well known that $\varepsilon$-expanded $\beta$-functions obtained in the massive renormalization scheme differ from those arising in massless renormalization already in the second order in $\varepsilon$, the same applies to the values of fixed points and other intermediate renormalization group functions (see [31] for instance). But of course critical exponents or other observables will not depend on the renormalization scheme (see below).

Let us pass on to study the expressions (4.3), (4.4) directly at fixed dimension. The usual way of dealing with $\beta$-functions of models containing several couplings obtained in this scheme consists in numerical solution of the system of equations for fixed points. Being asymptotic, the series in the coupling constants are represented in the form of the corresponding resummed expressions $\beta^{\text {res }}$ [44]. However, the numerical solution of the

Table 1. The fixed points of ternary solutions as obtained in the $\varepsilon^{3}$ expansion in the massless renormalization scheme [10]. $g_{G}^{*}, g_{U}^{*}, g_{S}^{*}$ are given by $(4.6),(4.7),(4.8)$.

$$
\begin{array}{llll}
G & g_{11}^{*}=0 & g_{22}^{*}=0 & g_{12}^{*}=g_{G}^{*} \\
U & g_{11}^{*}=g_{S}^{*} & g_{22}^{*}=0 & g_{12}^{*}=g_{U}^{*} \\
U^{\prime} & g_{11}^{*}=0 & g_{22}^{*}=g_{S}^{*} & g_{12}^{*}=g_{U}^{*} \\
S & g_{11}^{*}=g_{S}^{*} & g_{22}^{*}=g_{S}^{*} & g_{12}^{*}=g_{S}^{*}
\end{array}
$$




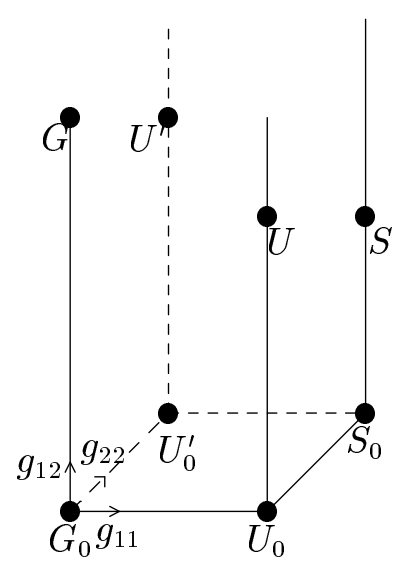

Figure 4. Fixed points of ternary polymer solution. $G_{0}, U_{0}, U_{0}^{\prime}, S_{0}$ correspond to mutually non-interacting chains, $G, U, U^{\prime}, S$ correspond to presence of mutually-avoiding interaction $\left(g_{12} \neq 0\right)$.

Table 2. Fixed points of ternary solutions as obtained in $\varepsilon^{2}$-expansion in the massive renormalization scheme. $v_{a b}$ are normalized couplings related to $g_{a b}$ by $v_{a b}=D_{2}^{m} g_{a b}$, where $D_{2}^{m}$ is the one-loop integral.

$$
\begin{array}{llll}
G & g_{11}^{*}=0 & g_{22}^{*}=0 & g_{12}^{*}=\frac{3 \varepsilon}{2}+\frac{3 \varepsilon^{2}}{2} \\
U & g_{11}^{*}=\frac{3 \varepsilon}{4}+\frac{111 \varepsilon^{2}}{128} & g_{22}^{*}=0 & g_{12}^{*}=\frac{9 \varepsilon}{8}+\frac{327 \varepsilon^{2}}{256} \\
U^{\prime} & g_{11}^{*}=0 & g_{22}^{*}=\frac{3 \varepsilon}{4}+\frac{111 \varepsilon^{2}}{128} & g_{12}^{*}=\frac{9 \varepsilon}{8}+\frac{327 \varepsilon^{2}}{256} \\
S & g_{11}^{*}=\frac{3 \varepsilon}{4}+\frac{111 \varepsilon^{2}}{128} & g_{22}^{*}=\frac{3 \varepsilon}{4}+\frac{111 \varepsilon^{2}}{128} & g_{12}^{*}=\frac{3 \varepsilon}{4}+\frac{111 \varepsilon^{2}}{128}
\end{array}
$$

resummed fixed point equation leads in our case to inconsistent results as will be shown in Sec. 6.. An alternative to this procedure and thus a third possibility to proceed was proposed by Nickel and is called a pseudo- $\varepsilon$ expansion[45]. As to our knowledge it was intensively exploited up to now only in theories with one coupling (see [37]), here we are going to use this 'third way' to deal with an expansion in several couplings. We introduce the "pseudo-epsilon" parameter $\tau$ into the expressions for the $\beta$-functions $\beta_{v_{a a}}^{m}, \beta_{v_{12}}^{m}$ in (4.3), (4.4) by:

$$
\begin{aligned}
& -\beta_{v_{a a}}^{m} /\left((4-d) v_{a a}\right)=\tau-\frac{4 v_{a a}}{3}+\ldots, \quad a=1,2, \\
& -\beta_{v_{12}}^{m} /\left((4-d) v_{12}\right)=\tau-\frac{1}{3}\left(v_{11}+v_{22}+2 v_{12}\right)+\ldots,
\end{aligned}
$$

and look for the fixed point solutions as series in $\tau$. The resulting series for the fixed points then can be either resummed (when the numerical value of the fixed points is needed) or they can be substituted into the other 
expansions arising in theory. In the final results we substitute $\tau=1$.

Performing this procedure we get the fixed point values as series in the pseudo-epsilon parameter $\tau$ up to the order $\tau^{3}$ : Performing this procedure we get the fixed point values as series in the pseudo-epsilon parameter $\tau$ up to the order $\tau^{3}$ :

$$
\begin{aligned}
& v_{G}=3 / 2 \tau+\left(3 i_{1}-3 / 2\right) \tau^{2}+\left(-\frac{9 i_{6}}{8}-\frac{9 i_{1}}{4}+3 / 8+12 i_{1}{ }^{2}-\frac{9 i_{4}}{2}-\right. \\
& \left.\frac{27 i_{5}}{8}-\frac{9 i_{7}}{8}\right) \tau^{3}, \\
& v_{U}=\frac{9 \tau}{8}+\left(\frac{93 i_{1}}{32}-\frac{93}{64}+\frac{3 i_{2}}{32}\right) \tau^{2}-\left(\frac{387 i_{6}}{512}+\frac{9 i_{8}}{64}+\frac{33 i_{2}}{64}+\frac{1281 i_{1}}{256}-\right. \\
& \frac{459}{512}+\frac{693 i_{7}}{512}+\frac{27 i_{3}}{128}+\frac{369 i_{4}}{64}+\frac{1485 i_{5}}{512}-\frac{3 i_{2}{ }^{2}}{64}-\frac{9 i_{2} i_{1}}{8}- \\
& \left.\frac{969 i_{1}^{2}}{64}-\frac{27 d i_{2}}{512}\right) \tau^{3}, \\
& v_{S}=3 / 4 \tau+\left(\frac{3 i_{2}}{16}+\frac{33 i_{1}}{16}-\frac{33}{32}\right) \tau^{2}+\left(3 / 4-\frac{27 i_{2}}{32}-\frac{261 i_{5}}{128}-\right. \\
& \frac{135 i_{4}}{32}-\frac{261 i_{1}}{64}-\frac{63 i_{6}}{128}+\frac{3 i_{2}{ }^{2}}{32}+\frac{33 i_{2} i_{1}}{16}+\frac{9 d i_{2}}{128}-\frac{99 i_{7}}{128}-\frac{9 i_{8}}{32}- \\
& \left.\frac{9 i_{3}}{32}+\frac{363 i_{1}{ }^{2}}{32}\right) \tau^{3} .
\end{aligned}
$$

Expressions (4.6) - (4.8) [10] and the expressions (4.3) - (4.13) obtained here give the fixed point values of ternary solutions in the massless and massive renormalization schemes and are the main results to be used in the subsequent calculations.

Looking for the stability of above described fixed points one finds that only the fixed point $S$ is stable and thus in the excluded volume limit of infinitely long chains the behavior of a system of two polymer species is described by the same scaling laws as a solution of only one polymer species. Nevertheless studying polymer mixtures at different temperatures and also taking into account that real polymer chains are not infinitely long one in fact may study crossover phenomena in the system which are governed by the unstable fixed points as well. The knowledge of the total flow allows for a description of the crossover phenomena in whole region [10].

However, for the purpose of our study we are interested only in the values of fixed points and properties of the star vertex functions in these fixed points.

\section{Results for Exponents}

For homogeneous stars of polymer chains of one species alone serveral sets of star exponents have been defined, each describing either the scaling properties of the configurational number (see formula (1.3) of this article), or the anomalous dimensions of star vertices, etc. Due to scaling relations these exponents can be expressed in terms of each other [2]. In this sense each set of star exponents forms a complete basis. For copolymer and MAW stars considered here we choose to present our results in terms of the exponents $\eta_{f_{1} f_{2}}$ and $\eta_{f}^{M A W}$ given by fixed point values of functions $\eta_{* f_{1} f_{2}}\left(g_{a b}\right)$ (3.13) and $\eta_{f}^{\mathrm{MAW}}\left(g_{a b}\right)$ (3.14). So let us define the asymptotic values of copolymer 
star exponents and MAW star exponents by:

$$
\begin{aligned}
\eta_{f_{1} f_{2}}^{S} & =\left.\eta_{* f_{1} f_{2}}\left(g_{a b}\right)\right|_{S}, \\
\eta_{f_{1} f_{2}}^{G} & =\left.\eta_{* f_{1} f_{2}}\left(g_{a b}\right)\right|_{G}, \\
\eta_{f_{1} f_{2}}^{U} & =\left.\eta_{* f_{1} f_{2}}\left(g_{a b}\right)\right|_{U}=\left.\eta_{* f_{2} f_{1}}\left(g_{a b}\right)\right|_{U^{\prime}}, \\
\eta_{f}^{\mathrm{MAW}} & =\left.\eta_{f}^{\mathrm{MAW}}\left(g_{a b}\right)\right|_{G} .
\end{aligned}
$$

In the case of the symmetric fixed point $S$ where we reproduce the results for homogeneous polymer stars [10] we can relate exponent $\eta_{f_{1} f_{2}}^{S}$ to configurational number star exponents $\gamma_{f}$ introduced by (1.3):

$$
\gamma_{f_{1} f_{2}}^{S}-1 \equiv \gamma_{f_{1}+f_{2}}-1=\nu \eta_{f_{1}, f_{2}}^{S}+(\nu(2-\eta)-1)\left(f_{1}+f_{2}\right)
$$

In the case of the fixed point $G$ (two sets of mutually avoiding random walks) substituting $\nu=1 / 2$ and $\eta=0$ to (5.5) one may define $\gamma_{f_{1} f_{2}}^{G}$ :

$$
\gamma_{f_{1} f_{2}}^{G}-1=\frac{\eta_{f_{1}, f_{2}}^{G}}{2}
$$

and the number of configurations $\mathcal{Z}_{f_{1} f_{2}}^{G}$ of the star of two sets of mutually avoiding chains will scale with the number of monomers like:

$$
Z_{f_{1} f_{2}}^{G} \sim e^{\mu N\left(f_{1}+f_{2}\right)} N^{\gamma_{f_{1} f_{2}}^{G}-1} .
$$

In the unsymmetric case, when one set of chains is selfavoiding and the other is not, the exponents $\gamma_{f_{1} f_{2}}$ are not well defined. This can be seen from the equation (5.5) as it is not clear what should be the value of $\nu$ in the first term of its right hand side. On the other hand, the scaling law of the number of configurations on the basis of number of monomers will also be ill-defined. The set of self-avoiding walks will fill much larger volume for the same number of monomers than the non-self-avoiding walks. These two sets will not interact along the full length of self-avoiding walks.

Now on the basis of the expressions for the fixed points given in the previous section and the relations (5.1) - (5.4) one can find the series for the appropriate star exponents. In the case of the $\epsilon$-expansion we obtain the following values of the exponents $\eta_{f_{1} f_{2}}$ :

$$
\begin{aligned}
& \eta_{f_{1} f_{2}}^{G}(\varepsilon)=-f_{1} f_{2} \frac{\varepsilon}{2}+f_{1} f_{2}\left(f_{2}-3+f_{1}\right) \frac{\varepsilon^{2}}{8}-f_{1} f_{2}\left(f_{2}-3+f_{1}\right)\left(f_{1}+\right. \\
& \left.f_{2}+3 \zeta(3)-3\right) \frac{\varepsilon^{3}}{16}, \\
& \eta_{f_{1} f_{2}}^{U}(\varepsilon)=f_{1}\left(1-f_{1}-3 f_{2}\right) \frac{\varepsilon}{8}+f_{1}\left(25-33 f_{1}+8 f_{1}{ }^{2}-91 f_{2}+42 f_{1} f_{2}+\right. \\
& \left.18 f_{2}{ }^{2}\right) \frac{\varepsilon^{2}}{256}+f_{1}\left(577-969 f_{1}+456 f_{1}{ }^{2}-64 f_{1}{ }^{3}-2463 f_{2}+2290 f_{1} f_{2}-\right. \\
& 492 f_{1}{ }^{2} f_{2}+1050 f_{2}{ }^{2}-504 f_{1} f_{2}{ }^{2}-108 f_{2}{ }^{3}-712 \zeta(3)+936 f_{1} \zeta(3)- \\
& \left.224 f_{1}{ }^{2} \zeta(3)+2652 f_{2} \zeta(3)-1188 f_{1} f_{2} \zeta(3)-540 f_{2}{ }^{2} \zeta(3)\right) \frac{\varepsilon^{3}}{4096},
\end{aligned}
$$




$$
\begin{aligned}
& \eta_{f_{1} f_{2}}^{M A W}(\varepsilon)=-\left(f_{1}-1\right) f_{1} \frac{\varepsilon}{4}+f_{1}\left(f_{1}-1\right)\left(2 f_{1}-5\right) \frac{\varepsilon^{2}}{16}-\left(f_{1}-1\right) f_{1}\left(4 f_{1}{ }^{2}-\right. \\
& \left.20 f_{1}+8 f_{1} \zeta(3)-19 \zeta(3)+25\right) \frac{\varepsilon^{3}}{32}
\end{aligned}
$$

In the case of the pseudo- $\varepsilon$ expansion obtained in the massive scheme our series for the $\eta_{f_{1} f_{2}}$ exponents read:

$$
\begin{aligned}
& \eta_{f_{1} f_{2}}^{G}(\tau)=(4-d)\left(-\frac{f_{1} f_{2}}{2} \tau+\frac{1}{2}\left(f_{2} f_{1}^{2}+f_{1} f_{2}^{2}-3 f_{1} f_{2}\right)\left(i_{1}-\frac{1}{2}\right) \tau^{2}+\right. \\
& \left.\left(\sum_{j=0}^{8} \eta_{G}^{j} i_{j}\right) \tau^{3}\right) \\
& \eta_{f_{1} f_{2}}^{U}(\tau)=(4-d)\left(\frac{1}{8}\left(-3 f_{1} f_{2}+f_{1}-f_{1}^{2}\right) \tau+\frac{1}{32}\left(\left(21 f_{2} f_{1}^{2}+9 f_{1} f_{2}^{2}-\right.\right.\right. \\
& \left.\left.46 f_{1} f_{2}+4 f_{1}^{3}+13 f_{1}-17 f_{1}^{2}\right)\left(i_{1}-\frac{1}{2}\right)+\left(f_{1}-f_{1}^{2}-f_{1} f_{2}\right) i_{2}\right) \tau^{2}+ \\
& \left.\left(\sum_{j=0}^{8} \eta_{U}^{j} i_{j}+\eta_{U}^{1,2} i_{1} i_{2}\right) \tau^{3}\right), \\
& \eta_{f_{1} f_{2}}^{M A W}(\tau)=(4-d)\left(-\frac{f(f-1)}{4} \tau+\frac{1}{4} f(f-1)(2 f-5)\left(i_{1}-\frac{1}{2}\right) \tau^{2}+\right. \\
& \left.\sum_{j=0}^{8} \eta_{M A W}^{j} i_{j} \tau^{3}\right) .
\end{aligned}
$$

The expressions for the three loop terms in (5.11) - (5.13) are given in the Appendix B. For the sake of completeness we give also the results in $\varepsilon$ - and pseudo- $\varepsilon$ expansions for the exponents $\nu$ and $\eta$ entering the relation (5.5):

$$
\begin{aligned}
& \eta(\varepsilon)=\frac{\varepsilon^{2}}{64}+\frac{17 \varepsilon^{3}}{1024} \\
& \nu(\varepsilon)=\frac{1}{2}+\frac{1}{16} \varepsilon+\frac{15}{512} \varepsilon^{2}+\left(\frac{135}{8192}-\frac{33 \zeta(3)}{1024}\right) \varepsilon^{3} \\
& \eta(\tau)=\frac{-(4-d)}{128} \tau\left(16 \tau i_{2}-12 \tau^{2} i_{2}-24 \tau^{2} i_{8}+8 \tau^{2} i_{2}^{2}+88 \tau^{2} i_{1} i_{2}\right)(5 \\
& \nu(\tau)=\frac{1}{2}+\frac{4-d}{16} \tau-\frac{(4-d)}{512}\left(4-40 i_{1}+8 i_{2}+4 d\right) \tau^{2}- \\
& \frac{(4-d)}{512}\left(6+12 i_{2}+10 i_{1}+84 i_{4}+63 i_{5}+3 i_{6}+33 i_{7}-12 i_{8}-\right. \\
& \left.220 i_{1}^{2}+4 i_{2}^{2}+24 i_{1} i_{2}-d+10 i_{1} d-2 i_{2} d-\frac{d^{2}}{2}\right) \tau^{3}
\end{aligned}
$$


Table 3. Values of the copolymer star exponent $\eta_{f_{1} f_{2}}$ obtained in the first $(\sim \varepsilon)$, second $\left(\sim \varepsilon^{2}\right)$ and third $\left(\sim \varepsilon^{3}\right)$ order in $\varepsilon$ in the Gaussian $(G)$ and unsymmetrical $(U)$ fixed points for different values of $f_{1}, f_{2}$ at $\varepsilon=1$. res stands for the results obtained by Padé-Borel resummation of $\varepsilon^{3}$-series.

\begin{tabular}{rrrrrrrrrr}
$f_{1}$ & $f_{2}$ & \multicolumn{3}{c}{ Fixed point $G$} & \multicolumn{4}{c}{ Fixed point $U$} \\
& & $\sim \varepsilon$ & $\sim \varepsilon^{2}$ & $\sim \varepsilon^{3}$ & res. & $\sim \varepsilon$ & $\sim \varepsilon^{2}$ & $\sim \varepsilon^{3}$ & res. \\
\hline & & & & & & & & & \\
1 & 1 & -.50 & -.63 & -.46 & -.56 & -.38 & -.50 & -.28 & -.43 \\
1 & 2 & -1.00 & -1.00 & -1.00 & & -.75 & -.85 & -.69 & -.80 \\
1 & 3 & -1.50 & -1.13 & -1.99 & -1.36 & -1.13 & -1.07 & -1.33 & -1.11 \\
2 & 1 & -1.00 & -1.00 & -1.00 & & -1.00 & -.98 & -.71 & -1.00 \\
2 & 2 & -2.00 & -1.50 & -2.65 & -1.81 & -1.75 & -1.37 & -2.37 & -1.62 \\
2 & 3 & -3.00 & -1.50 & -5.71 & -2.50 & -2.50 & -1.47 & -4.99 & -2.19 \\
3 & 1 & -1.50 & -1.13 & -1.99 & -1.36 & -1.88 & -1.28 & -1.70 & -1.50 \\
3 & 2 & -3.00 & -1.50 & -5.71 & -2.50 & -3.00 & -1.36 & -6.19 & -2.47 \\
3 & 3 & -4.50 & -1.13 & -12.27 & -3.48 & -4.13 & -1.02 & -12.83 & -3.26
\end{tabular}

\section{Resummation}

As is well known the series appearing in the perturbation theory of the field theoretic RG scheme are not convergent. Already the data given in tables 3 and 4 indicate such a behavior for the exponents $\eta_{f_{1} f_{2}}$, when the series is summed up without further analysis.

The growth of the coefficients of perturbation theory series may be estimated using information such as the growth of the combinatorial multiplicity of diagrams. It was proven that the series for the $\beta$ function of the $O(m)$ symmetric $\phi^{4}$ model with one coupling $g$ has the following asymptotic behavior $[46,47]$ :

$$
\begin{aligned}
\beta(g) & =\sum_{k} A_{k} g^{k} \\
A_{k} & =c k^{b_{0}}(-a)^{k} k ![1+O(1 / k)],, k \rightarrow \infty
\end{aligned}
$$

the quantities $a, b_{0}, c$ were calculated in $[46,48]$. Such a behavior is also expected and may be proven for the critical exponents as series in terms of the coupling. These results also show the divergence of the $\varepsilon$ and pseudo $\varepsilon$ expansions used here and indicate their Borel summability [49]. This procedure of resummation takes into account the asymptotic growth of the coefficients and allows to map the asymptotic series to a convergent series with the same limiting value. The function $\beta_{a a}$ considered here (3.11) coinsides with the $O(m)$ symmetric $\beta$ function (6.1) in the polymer limit $m=0$. So its asymptotic behavior is known from this study. The asymptotic behaviour of the off diagonal $\beta$ function $\beta_{12}$ was found by instanton analysis (see $[50,51])$ in $[10]$.

Let us introduce the techniques for resummation of the series based on the knowlege of the asymptotic behavior. Her we make use of Padé-Borel resummation and a resummation extended by a conformal mapping. The first way of resummation is applicable only for alternating series, while the second is more universal.

The resummation procedures are as follows. For the asymptotic series 
Table 4. Values of the copolymer star exponent $\eta_{f_{1} f_{2}}$ obtained in the one- $(\sim$ $\tau)$, two- $\left(\sim \tau^{2}\right)$ and three $\left(\sim \tau^{3}\right)$ loop approximation (power of $\tau$ corresponds to the number of loops) in $3 d$ theory in Gaussian $(G)$ and unsymmetrical $(U)$ fixed points for different values of $f_{1}, f_{2}$. res stands for the results obtained by Padé-Borel resummation of three-loop series.

\begin{tabular}{rrrrrrrrrr}
$f_{1}$ & $f_{2}$ & \multicolumn{4}{c}{ Fixed point $G$} & \multicolumn{4}{c}{ Fixed point $U$} \\
& & $\sim \tau$ & $\sim \tau^{2}$ & $\sim \tau^{3}$ & res. & $\sim \tau$ & $\sim \tau^{2}$ & $\sim \tau^{3}$ & res. \\
\hline & 1 & -.50 & -.58 & -.56 & -.57 & -.38 & -.46 & -.43 & -.44 \\
1 & 2 & -1.00 & -1.00 & -1.00 & & -.75 & -.82 & -.78 & -.80 \\
1 & 3 & -1.50 & -1.25 & -1.42 & -1.34 & -1.13 & -1.09 & -1.11 & -1.10 \\
2 & 1 & -1.00 & -1.00 & -1.00 & & -1.00 & -.99 & -.98 & -.99 \\
2 & 2 & -2.00 & -1.67 & -1.93 & -1.80 & -1.75 & -1.50 & -1.71 & -1.60 \\
2 & 3 & -3.00 & -2.00 & -3.01 & -2.45 & -2.50 & -1.82 & -2.56 & -2.13 \\
3 & 1 & -1.50 & -1.25 & -1.42 & -1.34 & -1.88 & -1.48 & -1.82 & -1.64 \\
3 & 2 & -3.00 & -2.00 & -3.01 & -2.45 & -3.00 & -1.91 & -3.18 & -2.43 \\
3 & 3 & -4.50 & -2.25 & -5.09 & -3.37 & -4.13 & -2.06 & -4.97 & -3.14
\end{tabular}

for an exponent $\gamma$ given as a series in the expansion parameter $\varepsilon$

$$
\gamma(\varepsilon)=\sum_{j} \gamma^{(j)} \varepsilon^{j}
$$

one defines the Borel-Leroy transform $\gamma^{B}(\varepsilon)$ of the series by:

$$
\gamma^{B}(\varepsilon)=\sum_{j} \frac{\gamma^{(j)}}{\Gamma(j+b+1)}(\varepsilon)^{j},
$$

( $b$ being the fit parameter). Then the value of the initial series may be calculated from

$$
\gamma(\varepsilon)=\int_{0}^{\infty} d t t^{b} e^{-t} \gamma^{B}(\varepsilon t)
$$

Evaluating this for the truncated series as calculated from the perturbation theory and substituting instead of $\gamma^{B}(\varepsilon t)$ in (6.5) its analytic continuation in the form of Padé approximant this procedure constitutes the Padé Borel resummation.

The conformal mapping technique postulates in addition the knowledge of the constant $a$ entering (6.2). Assuming the behavior (6.2) holds also for the expansion of $\gamma(\varepsilon)$ in $\varepsilon$, one concludes that the singularity of the transformed series $\gamma^{B}(\varepsilon)$ closest to the origin is located at the point $(-1 / a)$ and one can map the $\varepsilon$ plane onto a circle with a mapping leaving the origin invariant:

$$
w=\frac{(1+a \varepsilon)^{1 / 2}-1}{(1+a \varepsilon)^{1 / 2}+1}, \quad \varepsilon=\frac{4}{a} \frac{w}{(1-w)^{2}} .
$$

Thus one obtains an expression for $\gamma^{B}(\varepsilon)$ convergent in the whole cut plane and, as a result, the expression for the resummed function $\gamma^{\text {res }}$. In order to weaken a possible singularity on $w$-plane the corresponding expression is multiplied by $(1-w)^{\alpha}$ and thus one more parameter $\alpha$ is introduced. In 
the resummation procedure the value of $a$ is taken from the known largeorder behavior of $\varepsilon$-expansion series while $\alpha$ was chosen in our calculations as a fit parameter defined by the condition of minimal difference between resummed 2nd order and 3rd order results. The resummation procedure was seen to be quite insensitive to the parameter $b$ introduced by the Borel-Leroy transformation (6.4).

For the resummation of the rexponents $\eta_{f_{1} f_{2}}$ we take into account in addition the knowledge of the combinatorial factors which multiply each contribution according to the numbers of chains $f_{1}$ and $f_{2}$. This leads to an additional factor $\left(f_{1}+f_{2}\right)^{k}$ for the $k$ th order contributions. It is taken into account by multiplying the constant $a$ by $\left(f_{1}+f_{2}\right)$. For resummation of the series at the fixed points $\mathrm{S}, \mathrm{G}$ and $\mathrm{U}$ the following values of $a=a^{\mathrm{S}}, a^{\mathrm{G}}, a^{\mathrm{U}}$ are used $[46,10]$ :

$$
a^{\mathrm{S}}=a^{\mathrm{G}}=3 / 8 \quad \text { and } \quad a^{\mathrm{U}}=27 / 64
$$

By analogy we use the same procedures as developed for the $\varepsilon$ expansion also for the $\tau$-expansion which we assume to have the same asymptotic behavior as it is in the same way collecting contributions of the same loop order.

Let us note here that the conventional resummation of the $\beta$ - functions in the massive approach leads to a severe inconsistency which is the reason for us to take the pseudo- $\varepsilon$ or $\tau$-expansion method.

The distinct feature of the $\beta_{a b}$-functions introduced here is that they are functions of different numbers of variables which leads to ambiguities in their analytical continuation via Padé approximants or rational approximants of several variables (see [52]). Let us illustrate this on the example of the two-loop approximation, when the corresponding expressions read:

$$
\begin{aligned}
& \beta_{v_{a a}}=-(4-d) v_{a a} f_{v_{a a}}\left(v_{a a}\right), a=1,2, \\
& \beta_{v_{12}}=-(4-d) v_{12} f_{v_{12}}\left(v_{11}, v_{22}, v_{12}\right)
\end{aligned}
$$

with obvious expressions for $f_{v_{11}}, f_{v_{22}}, f_{v_{12}}$.

Now in order to obtain the analytical continuation of the Borel transformed functions one one variable $f_{v_{a a}}\left(v_{a a}\right)(6.7)$ one can make use of the [1/1] Padé approximant. Solving the correponding non linear equations numerically we get for the non-trivial fixed point $S: v_{11}=v_{22}=1.1857$ [53]. In order to apply a similar resummation technique to the function $f_{v_{12}}\left(v_{11}, v_{22}, v_{12}\right)(6.8)$ one can make use of a generalization of Padé approximants to the case of several variables, i.e. represent the Borel transform $F_{v_{12}}$ of $f_{v_{12}}$ in the form of a rational approximant of three variables:

$$
F_{v_{12}}^{P}\left(v_{11} t, v_{22} t, v_{12} t\right)=\frac{1+a_{1}\left(v_{11}, v_{22}, v_{12}\right) t+a_{2}\left(v_{11}, v_{22}, v_{12}\right) t^{2}}{1+b\left(v_{11}, v_{22}, v_{12}\right) t}
$$

In spite of the fact that the rational approximant (6.9) holds projection properties of the initial series (6.8), i. e. putting any pair of variables $\left\{v_{11}, v_{22}, v_{12}\right\}$ to zero in (6.9) one gets the appropriate [1/1] Padé approximant for the remaining variable, the "global" symmetry is now not preserved. Due to different analytical continuations for the Borel transforms of the series (6.7) on one hand and (6.8) on the other, solving the fixed-point equation

$$
f_{v_{12}}^{r e s}=0
$$

we will never obtain a symmetrical solution $v_{11}^{*}=v_{22}^{*}=v_{12}^{*} \neq 0$. Really, substituting the solution $v_{11}^{*}=v_{22}^{*}=0$ into the equation (6.10) and solving 
it numerically we get for fixed point $G: v_{G}=2.1112$. For the fixed points $U$ and $U^{\prime}$ the corresponding value of $v_{12}$ equals: $v_{U}=1.6955$. For the case $S$ substituting into the equation (6.10) $v_{11}=v_{22}=1.1857$ instead getting "symmetrical" solution $v_{11}^{*}=v_{22}^{*}=v_{12}^{*} \neq 0$ we get $v_{12}=0.9571$ (!) [55]. The reason is that substituting numerical values of fixed point coordinates $v_{11}, v_{22}$ into (6.10) we loose information about contributions into fixed point value from different separate orders of the perturbation theory series.

So it appears quite natural to restore this information by generalizing the pseudo- $\varepsilon$ expansion [45] to the case of several couplings as described in section 4 .

\section{Numerical Results}

In this chapter we give results for the exponents $\eta_{f_{1} f_{2}}^{\mathrm{G}}, \eta_{f_{1} f_{2}}^{\mathrm{U}}$ and $\eta_{f}^{M A W}$. The exponent $\eta_{f_{1} f_{2}}^{\mathrm{S}}$ in the symmetrical fixed point $S$ describes a uniform star and coincides with $\eta_{f_{1}+f_{2}}$ defined in this context [2] (see formulas (1.3),(5.5) of this article as well). Results for the symmetrical case are to be found in $\varepsilon$-expansion in [13] and in pseudo- $\varepsilon$ expansion in [56].

\section{1. $d=3$}

Let us consider first the case $d=3$. Applying the Padé-Borel resummation technique one obtains the results given in the tables 3 and 4 for the $\varepsilon$ - and $\tau$ expansions in comparison with non-resummed results.

Even though the non-resummed results differ to great extend for higher numbers of arms resumation shows the consistency of the approaches on the numerical level. Further applying the resummation procedure based on the conformal mapping technique as described in the previous section we get the results given in tables 56 .

Table 5 . Values of the copolymer star exponent $\eta_{f_{1} f_{2}}^{G}$ at $d=3$ obtained by $\varepsilon$-expansion $\left(\eta_{\varepsilon}^{G}\right)$ and by fixed dimension technique $\left(\eta_{3 d}^{G}\right)$.

\begin{tabular}{llllllll}
$f_{1}$ & $f_{2}$ & $\eta_{\varepsilon}^{G}$ & $\eta_{3 d}^{G}$ & $f_{1}$ & $f_{2}$ & $\eta_{\varepsilon}^{G}$ & $\eta_{3 d}^{G}$ \\
\hline 1 & 1 & -0.56 & -0.58 & 3 & 3 & -3.38 & -3.57 \\
1 & 2 & -0.99 & -1.00 & 3 & 4 & -4.21 & -4.50 \\
1 & 3 & -1.33 & -1.35 & 3 & 5 & -4.94 & -5.36 \\
1 & 4 & -1.63 & -1.69 & 3 & 6 & -5.62 & -6.15 \\
1 & 5 & -1.88 & -1.98 & 4 & 4 & -5.27 & -5.71 \\
1 & 6 & -2.10 & -2.24 & 4 & 5 & -6.24 & -6.84 \\
2 & 2 & -1.77 & -1.81 & 4 & 6 & -7.12 & -7.90 \\
2 & 3 & -2.45 & -2.53 & 5 & 5 & -7.42 & -8.24 \\
2 & 4 & -3.01 & -3.17 & 5 & 6 & -8.50 & -9.54 \\
2 & 5 & -3.51 & -3.75 & 6 & 6 & -9.78 & -11.07 \\
2 & 6 & -3.95 & -4.28 & & & &
\end{tabular}

Comparing the numerical values listed in the above tables it is convincing that the two approaches and the different resummation procedures all lead to results which lie within a bandwidth of consistency, which is broadening for larger values of number of chains. This is not surprising as we have seen in section 5. that our expansion parameters are multiplied by the number of chains. Rather it is remarkable that even for a total number of chains of 
Table 6 . Values of the copolymer star exponent $\eta_{f_{1} f_{2}}^{U}$ at $d=3$ obtained by $\varepsilon$-expansion $\left(\eta_{\varepsilon}^{U}\right)$ and by fixed dimension technique $\left(\eta_{3 d}^{U}\right)$.

\begin{tabular}{llllllll}
$f_{1}$ & $f_{2}$ & $\eta_{\varepsilon}^{U}$ & $\eta_{3 d}^{U}$ & $f_{1}$ & $f_{2}$ & $\eta_{\varepsilon}^{U}$ & $\eta_{3 d}^{U}$ \\
\hline 1 & 1 & -0.43 & -0.45 & 4 & 1 & -2.39 & -2.47 \\
1 & 2 & -0.79 & -0.81 & 4 & 2 & -3.33 & -3.50 \\
1 & 3 & -1.09 & -1.09 & 4 & 3 & -4.20 & -4.48 \\
1 & 4 & -1.35 & -1.37 & 4 & 4 & -5.02 & -5.40 \\
1 & 5 & -1.60 & -1.64 & 4 & 5 & -5.80 & -6.30 \\
1 & 6 & -1.81 & -1.89 & 4 & 6 & -6.53 & -7.15 \\
2 & 1 & -0.98 & -0.98 & 5 & 1 & -3.21 & -3.38 \\
2 & 2 & -1.58 & -1.60 & 5 & 2 & -4.28 & -4.57 \\
2 & 3 & -2.13 & -2.19 & 5 & 3 & -5.28 & -5.71 \\
2 & 4 & -2.61 & -2.71 & 5 & 4 & -6.24 & -6.81 \\
2 & 5 & -3.05 & -3.21 & 5 & 5 & -7.15 & -7.89 \\
2 & 6 & -3.46 & -3.68 & 5 & 6 & -8.02 & -8.92 \\
3 & 1 & -1.64 & -1.67 & 6 & 1 & -4.11 & -4.40 \\
3 & 2 & -2.44 & -2.52 & 6 & 2 & -5.29 & -5.73 \\
3 & 3 & -3.16 & -3.30 & 6 & 3 & -6.41 & -7.03 \\
3 & 4 & -3.82 & -4.04 & 6 & 4 & -7.48 & -8.28 \\
3 & 5 & -4.44 & -4.75 & 6 & 5 & -8.51 & -9.50 \\
3 & 6 & -5.01 & -5.42 & 6 & 6 & -9.50 & -10.69
\end{tabular}

the order of 10 (see tables 5,6 ) we still receive results which are comparable to each other.

Also it seems remarkable that at least for low numbers of chains $\left(f_{1}+\right.$ $\left.f_{2} \sim 4\right)$ the non resummed $\tau$-expansion seems to give results which do not differ essentially from the resummed values. However, applying the conformal mapping technique to the series in $\tau$-expansion does not improve the Padé-Borel results.

7.2. $d=2$

While star polymers up to now have not found an experimental realization in two dimensions, their study is of some theoretical interest. It has been shown that the scaling dimensions of two-dimensional uniform polymer stars belong to a limiting case of the so-called conformal Kac table [57-59]. They have also been calculated exactly by Couloumb gas techniques $[12,17]$. An exact relation has also been proposed for stars of mutually avoiding walks [29]. But it is still an open question if exact results for the copolymer star system may be derived in this formalism.

Our numerical results for the exponents $\eta_{f_{1} f_{2}}^{\mathrm{G}}, \eta_{f_{1} f_{2}}^{\mathrm{U}}, \eta_{f_{1} f_{2}}^{\mathrm{MAW}}$ are presented in tables $8,9,7$.

Exact results for exponents of two dimensional systems which are described by a conformal field theory with central charge $c<1$ may be taken from the Kac table of scaling dimensions:

$$
h_{p, q}(m)=\frac{[(m+1) p-m q]^{2}-1}{4 m(m+1)}
$$

where $p, q$ are integers in the minimal block

$$
1 \leq p \leq m-1, \quad 1 \leq q \leq p
$$


Table 7. Values of $\eta_{f}^{M A W}$ exponents of star of mutually avoiding walks at $d=2, d=3$ obtained by $\varepsilon$-expansion $\left(\eta_{\varepsilon}^{M A W}\right)$ and by fixed dimension technique $\left(\eta_{3 d}^{M A W}, \eta_{2 d}^{M A W}\right)$. The last column gives the exact conjecture $\eta_{\text {exact }}^{M A W}$ at $d=2[29]$.

\begin{tabular}{llllll} 
& \multicolumn{2}{c}{$d=3$} & \multicolumn{3}{c}{$d=2$} \\
$f$ & $\eta_{\varepsilon}^{M A W}$ & $\eta_{3 d}^{M A W}$ & $\eta_{\varepsilon}^{M A W}$ & $\eta_{2 d}^{M A W}$ & $\eta_{\text {exact }}^{M A W}$ \\
\hline 1 & 0 & 0 & 0 & 0 & -.250 \\
2 & -.56 & -.56 & -1.20 & -1.19 & -1.250 \\
3 & -1.38 & -1.36 & -2.71 & -2.60 & $-2.916(6)$ \\
4 & -2.36 & -2.34 & -4.36 & -4.07 & -5.250 \\
5 & -3.43 & -3.43 & -6.04 & -5.61 & -8.250 \\
6 & -4.58 & -4.64 & -7.78 & -7.17 & $-11.916(6)$ \\
7 & -5.81 & -5.93 & -9.57 & -8.75 & -16.250 \\
8 & -7.09 & -7.30 & -11.42 & -10.36 & -21.250 \\
9 & -8.42 & -8.74 & -13.31 & -11.97 & $-26.916(6)$ \\
10 & -9.81 & -10.24 & -15.25 & -13.60 & -33.250
\end{tabular}

Table 8. Values of the copolymer star exponent $\eta_{f_{1} f_{2}}^{G}$ at $d=2$ obtained by $\varepsilon$-expansion $\left(\eta_{\varepsilon}^{G}\right)$ and by fixed dimension technique $\left(\eta_{2 d}^{G}\right)$.

\begin{tabular}{llllllll}
$f_{1}$ & $f_{2}$ & $\eta_{\varepsilon}^{G}$ & $\eta_{3 d}^{G}$ & $f_{1}$ & $f_{2}$ & $\eta_{\varepsilon}^{G}$ & $\eta_{3 d}^{G}$ \\
\hline 1 & 1 & -1.20 & -1.22 & 3 & 3 & -6.05 & -6.23 \\
1 & 2 & -1.98 & -1.98 & 3 & 4 & -7.36 & -7.63 \\
1 & 3 & -2.56 & -2.58 & 3 & 5 & -8.49 & -8.84 \\
1 & 4 & -2.99 & -3.04 & 3 & 6 & -9.50 & -9.91 \\
1 & 5 & -3.36 & -3.43 & 4 & 4 & -9.06 & -9.44 \\
1 & 6 & -3.68 & -3.78 & 4 & 5 & -10.55 & -11.03 \\
2 & 2 & -3.41 & -3.45 & 4 & 6 & -11.89 & -12.45 \\
2 & 3 & -4.49 & -4.59 & 5 & 5 & -12.38 & -12.98 \\
2 & 4 & -5.37 & -5.52 & 5 & 6 & -14.03 & -14.74 \\
2 & 5 & -6.13 & -6.34 & 6 & 6 & -15.99 & -16.81 \\
2 & 6 & -6.80 & -7.04 & & & &
\end{tabular}


Table 9. Values of the copolymer star exponent $\eta_{f_{1} f_{2}}^{U}$ at $d=2$ obtained by $\varepsilon$-expansion $\left(\eta_{\varepsilon}^{U}\right)$ and by fixed dimension technique $\left(\eta_{2 d}^{U}\right)$.

\begin{tabular}{llllllll}
$f_{1}$ & $f_{2}$ & $\eta_{\varepsilon}^{U}$ & $\eta_{3 d}^{U}$ & $f_{1}$ & $f_{2}$ & $\eta_{\varepsilon}^{U}$ & $\eta_{3 d}^{U}$ \\
\hline 1 & 1 & -.91 & -.96 & 4 & 1 & -4.35 & -4.41 \\
1 & 2 & -1.62 & -1.63 & 4 & 2 & -5.94 & -6.06 \\
1 & 3 & -2.16 & -2.16 & 4 & 3 & -7.39 & -7.57 \\
1 & 4 & -2.60 & -2.64 & 4 & 4 & -8.72 & -8.97 \\
1 & 5 & -3.00 & -3.03 & 4 & 5 & -9.96 & -10.27 \\
1 & 6 & -3.34 & -3.39 & 4 & 6 & -11.12 & -11.49 \\
2 & 1 & -1.94 & -1.96 & 5 & 1 & -5.71 & -5.83 \\
2 & 2 & -3.05 & -3.09 & 5 & 2 & -7.46 & -7.64 \\
2 & 3 & -4.00 & -4.04 & 5 & 3 & -9.09 & -9.35 \\
2 & 4 & -4.80 & -4.88 & 5 & 4 & -10.61 & -10.95 \\
2 & 5 & -5.52 & -5.63 & 5 & 5 & -12.06 & -12.47 \\
2 & 6 & -6.17 & -6.33 & 5 & 6 & -13.43 & -13.91 \\
3 & 1 & -3.11 & -3.13 & 6 & 1 & -7.17 & -7.35 \\
3 & 2 & -4.49 & -4.54 & 6 & 2 & -9.04 & -9.30 \\
3 & 3 & -5.70 & -5.80 & 6 & 3 & -10.82 & -11.17 \\
3 & 4 & -6.79 & -6.96 & 6 & 4 & -12.52 & -12.95 \\
3 & 5 & -7.81 & -8.01 & 6 & 5 & -14.14 & -14.65 \\
3 & 6 & -8.73 & -8.99 & 6 & 6 & -15.69 & -16.27
\end{tabular}

and $m$ is connected with the central charge $c$ by

$$
c=1-6 / m(m+1), \quad m \geq 3 .
$$

The exact result for the star exponents of uniform stars in two dimensions is received in the limiting case of $m=2$ (which means $c=0$ ) for half integer values of $p$ :

$$
x_{f}=2 h_{f / 2,0}=\left(9 f^{2}-4\right) / 48 .
$$

The scaling dimension $x_{f}$ is related to the exponent $\eta_{f}$ by:

$$
x_{f}=\frac{1}{2} F(d-2+\eta)-\eta_{f}
$$

For the exponents of the star of MAW the following result was conjectured for $d=2[29]$ :

$$
\eta_{f}^{\mathrm{MAW}}=x_{f}^{\mathrm{MAW}}=2 h_{0, f}=\frac{1-4 f^{2}}{12}
$$

These values are shown in the last column of table 7 . Plotting the resummed data for $\eta_{f}^{\mathrm{MAW}}$ from table 7 with respect to $f^{2}$ one finds good agreement with the conjectured slope of $-1 / 3$.

The qualitative behavior of the exponent $\eta_{f_{1} f_{2}}^{G}$ in the Gaussian fixed point is shown in Fig. 5. The steps in the 'flying carpet' correspond to the difference of the results of the two RG approaches. Note that the curvature of the surface along the diagonal in the $f_{1}, f_{2}$ plane has opposite sign to that along each of the axes. ¿From this curvature it is obvious that the dependence of the exponent on $f_{1}, f_{2}$ may not be described by a simple second order polynomial. The best fit we could find to our resummed data 


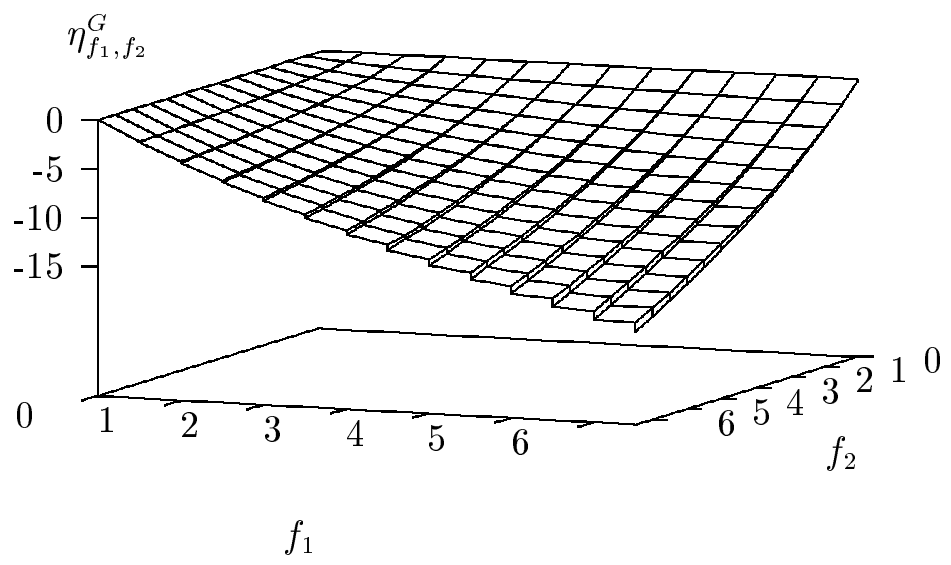

Figure 5. Exponent $\eta_{f_{1} f_{2}}^{G}$ in the 'Gaussian' fixed point at $d=2$ obtained in $\varepsilon$-expansion and in fixed $d$ scheme. Steps on the "flying carpet" correspond to the difference of the results of the two renormalization group approaches.

using a simple formula which reproduces the exact results for $f_{1}+f_{2}=3$ reads:

$$
\eta_{f_{1} f_{2}}^{G, a p p}=-f_{1} f_{2}\left(a+b /\left(f_{1}+f_{2}\right)\right), \quad a=1 / 4, \quad b=3(1-a) .
$$

Note that the right hand side of (7.7) vanishes if $f_{1}$ or $f_{1}$ is zero according to our perturbative results. This might be a defect of the perturbation theory as a finite result may be expected in $d=2$ as in equations (7.4), (7.6) evaluated for $f=0$.

\section{Conclusions and Outlook}

We have extensively studied the spectrum of exponents governing the scaling properties of stars of walks taking into account the self and mutual interactions of a system of two species of polymers. This study was performed in the frames of field theoretical RG theory using two complementary approaches: The renormalization at zero mass in conjunction with $\varepsilon$ expansion and massive renormalization at fixed dimension with numerical evaluation of loop integrals. We have formulated the problem of finding the scaling exponents of stars of interacting and non interacting walks in terms of the determination of the scaling dimensions of composite field operators of Lagrangean field theory. On the one hand this allows for the application of well developped formalisms and methods for analysing the scaling properties. On the other hand this defines these new families of exponents which extends previous sets in the framework of Lagrangean field theory. Our results agree with the previous studies of special cases which were in part done only to 2 nd order of the $\varepsilon$ expansion [29]. We have here considered the general case of a star of two mutually avoiding sets of walks, the walks of each set either interacting or not. Also we have studied the case of a star of mutually interacting walks. All calculations were performed to third order of perturbation theory. The set of exponents are given in $\varepsilon$-expansion 
(formulas (5.8) - (5.10)) and the results of the massive approach are given in terms of a pseudo- $\varepsilon$-expansion $(5.11)$ - (5.13)). The latter has proven to be a most suitable tool to evaluate this massive theory containing serveral couplings. We have shown that the convetional way of direct solution even of the resummed expressions for the fixed points of the theory would lead to severe problems in this case. We have evaluated the series obtained in both approaches for space dimensionality $d=2$ and $d=3$. Numerical values are produced by careful resummation of the asymptotic series using the results of an instanton analysis of the three coupling problem [10]. For comparison we have also given the results of naive summation as well as standard Padé Borel resummation for selected cases.

We have found remarkable consistency and stability of the results in $d=$ 2 and $d=3$ with expected growing of deviations for large number of arms of one star. The same methods were applied previously to the problem of uniform star polymers and have led to results $[13,56]$ in good agreement with Monte Carlo (MC) simulations [18-20]. We hope our present calculations might also stimulate MC studies of the copolymer star problem.

The study we performed for two dimenensions might have no direct application to the physics of real polymers but it could perhaps give some insight to the problem of mapping our theory to a two dimensional conformal field theory. The resummed values of the exponents for stars of mutually avoiding walks are in fair agreement with an exact result previously conjectured [29]. The exponents for the case of stars of two mutually avoiding sets of walks on the other hand show a dependence on the numbers of walks which may not be described by a second order polynomial as derived from the general Kac formula [57-59]. This may be seen already qualitively from the fact that the curvature of the function $\eta_{f_{1} f_{2}}$ (see Fig. 5) of the two variables $f_{1}, f_{2}$ along each of the axes in the $f_{1} f_{2^{-}}$plane has the opposite sign to the curvature along the diagonal $f_{1}=f_{2}$. It is this fact on the other hand, which shows that the series of exponents $\left(\eta_{f_{1} f_{2}}\right)_{f_{2}}$ with $f_{1}$ fixed is a good candidate for finding its application in the theory of multifractal (MF) spectra [60]. The MF spectrum describing the moments of a fractal probability measure fulfills exact conditions of convexity. Deriving such a MF spectrum on the other hand from the scaling dimensions of a series of composite field operators is only possible if the scaling dimensions show the appropriate convexity [61]. This in fact is given for our case and the series of exponents may be related to the MF spectrum generated by harmonic diffusion near an absorbing fractal [21]. This relation and the calculation of the MF spectrum on the basis of the results presented here, is subject of a separate publication [22].

\section{Acknowledgements}

This work has profited from useful discussions with Lothar Schäfer, Essen. It was supported in part by SFB 237 of Deutsche Forschungsgemeinschaft and by the Ukrainian Fundation of Fundamental Studies (grant No 24/173). C.v.F. acknowledges hospitality at the School of Physics, Tel Aviv University and support by a Minerva fellowship.

\section{A Loop Integrals. Graphs, $\varepsilon$-expansion, Numerical Values}

This appendix is devoted to the contributions to perturbation theory, their representation in terms of Feynman graphs and corresponding loop integrals, and the evaluation of these integrals for the two RG approaches. Fig. 6 
shows the Feynman graphs up to the three loop order representing the contributions to the functions $\Gamma^{(2)}$, and $\Gamma^{(4)}$ (we keep labeling of [42]). As it was noted in the section 2 . the vertex function $\Gamma^{(* f)}$ of homogeneous star (2.12) can be determined as the $m=0$ component limit of product of $f$ fields $\phi^{(a)}$ with traceless symmetry reflected by a tensor $N^{a_{1} \ldots a_{f}}(2.13)$. It is easy to see that only graphs with one $N^{a_{1} \ldots a_{f}} \phi^{\left(a_{1}\right)} \ldots \phi^{\left(a_{f}\right)}$ insertion give nonzero contribution to $\Gamma^{(* f)}$ : the rest will contain some trace of $N^{a_{1} \ldots a_{f}}$ and thus will vanish. Relevant graphs can be obtained from the usual four-point graphs 2-U2 - 12-U4 by considering each vertex in turn to be of traceless symmetry. In the three-loop approximation considered here in $\Gamma^{(* f)}$ there appear two more graphs which can not be produced in this manner. They are labelled as 13 and 14 . In table 10 we show the correspondence between the numerical values of the loop integrals and appropriate Feynman graphs.

A diagramm with $L$ loops is to be multiplied by $S^{L}$ with

$$
S=2 \pi^{d / 2} \frac{1}{(2 \pi)^{d} \Gamma(d / 2)},
$$

but this renormalization factor can be absorbed into redefinition of the coupling constants $g_{a b} \rightarrow g_{a b} / S$.

Table 10. Values of the loop integrals (for graphs 2-M1 - 5-S3 for two-point function $\Gamma^{(2)}(k)$ the corresponding derivative $\partial / \partial k^{2}$ is given.

\begin{tabular}{llllll} 
Graph & $\begin{array}{l}\text { Integral } \\
\text { value }\end{array}$ & Graph & $\begin{array}{l}\text { Integral } \\
\text { value }\end{array}$ & Graph & $\begin{array}{l}\text { Integral } \\
\text { value }\end{array}$ \\
\hline 2-U2 & $D_{2}$ & 8 -U4 & $I_{4}$ & 14 & $I_{1} D_{2}$ \\
$3-\mathrm{U} 3$ & $D_{2}^{2}$ & 9 -U4 & $I_{5}$ & $2-\mathrm{M} 1$ & 0 \\
$4-\mathrm{U} 3$ & $I_{1}$ & $10-\mathrm{U}$ & $I_{6}$ & $3-\mathrm{S} 2$ & $I_{2}$ \\
$5-\mathrm{U} 4$ & $D_{2}^{3}$ & $11-\mathrm{U} 4$ & $I_{5}$ & $4-\mathrm{M} 3$ & 0 \\
$6-\mathrm{U} 4$ & $I_{1} D_{2}$ & $12-\mathrm{U} 4$ & $I_{7}$ & $5-\mathrm{S} 3$ & $I_{8}$ \\
$7-\mathrm{U} 4$ & $I_{3}$ & 13 & $D_{2}^{3}$ & &
\end{tabular}

In massless renormalization scheme loop integrals corresponding to these graphs are evaluated by $\varepsilon$-expansion at zero mass and non-zero external momenta chosen at certain point, called symmetry point, which is important from the technical point of view. The expressions read:

$$
\begin{aligned}
& D_{2}^{\varepsilon}=\frac{1}{\varepsilon}\left(1+\frac{\varepsilon}{2}+\frac{\varepsilon^{2}}{2}\right), \quad I_{1}^{\varepsilon}=\frac{1}{2 \varepsilon^{2}}\left(1+\frac{3 \varepsilon}{2}+\frac{5 \varepsilon^{2}}{2}-\frac{J \varepsilon^{2}}{2}\right), \\
& I_{2}^{\varepsilon}=-\frac{1}{8 \varepsilon}\left(1+\frac{5 \varepsilon}{4}\right), \quad I_{3}^{\varepsilon}=-\frac{1}{24 \varepsilon^{2}}\left(1+\frac{15 \varepsilon}{4}\right), \\
& I_{4}^{\varepsilon}=\frac{1}{6 \varepsilon^{3}}\left(1+3 \varepsilon+\frac{31 \varepsilon^{2}}{4}-\frac{3 J \varepsilon^{2}}{2}\right), \\
& I_{5}^{\varepsilon}=\frac{1}{3 \varepsilon^{3}}\left(1+\frac{5 \varepsilon}{2}+\frac{23 \varepsilon^{2}}{4}-\frac{3 J \varepsilon^{2}}{2}\right), \quad I_{6}^{\varepsilon}=\frac{1}{3 \varepsilon^{3}}\left(1+2 \varepsilon+\frac{13 \varepsilon^{2}}{4}\right), \\
& I_{7}^{\varepsilon}=\frac{\zeta(3)}{2 \varepsilon}, \quad I_{8}^{\varepsilon}=-\frac{1}{6 \varepsilon^{2}}(1+2 \varepsilon)
\end{aligned}
$$

Here the values of derivatives $\partial / \partial k^{2}$ of the function $\Gamma^{(2)}(k)$ are given at the point $k^{2}=1$. 
Table 11. Numerical values of normalized loop integrals $i_{j}$ calculated in the massive field theory framework $[42,43]$.

$\begin{array}{lll} & d=2 & d=3 \\ i_{1} & .781302412896 & .6666666667 \\ i_{2} & -.114635746230 & -.0740740741 \\ i_{3} & -.044703881514 & -.0376820725 \\ i_{4} & .569829439192 & .3835760966 \\ i_{5} & .659043562065 & .5194312413 \\ i_{6} & .650899895132 & .5000000000 \\ i_{7} & .40068563 & .1739006107 \\ i_{8} & -.157398409771 & -.0946514319\end{array}$

In the massive renormalization scheme loop integrals are calculated at non-zero mass and zero external momenta (to distinguish from (A.1) we will label them by " $m$ "). The mass - renormalization introduces a higher order correction to the propagator, which has to be taken into account in our calculation only in the first order term:

$$
D_{2}^{a_{1} a_{2}}=D_{2}+\frac{1}{9} I_{2} D_{21}\left(u_{a_{1} a_{1}}^{2}+u_{a_{2} a_{2}}^{2}\right)
$$

Here $D_{21}=(4-d) / 4$. This value has been substituted into the results for the beta functions and fixed points. $D_{21}$ does not enter expressions which are independent of the RG scheme, such as the resulting exponents. The integrals can be either $\varepsilon$-expanded (see formulas (4.9) from this article for instance) or numerically calculated at arbitrary space dimensions $[42,43]$. In particular for dimension $d=2$ and $d=3$ they are given in Table 11 with the following normalization:

$$
i_{1}=I_{1}^{m} /\left(D_{2}^{m}\right)^{2}, \quad i_{2}=I_{2}^{m} /\left(D_{2}^{m}\right)^{2}, \quad i_{j}=I_{j}^{m} /\left(D_{2}^{m}\right)^{3}, \quad j=3 \ldots 8 .
$$

Note that here values of the derivative $\partial / \partial k^{2}$ of the function $\Gamma^{(2)}(k)$ are given at $k^{2}=0$.

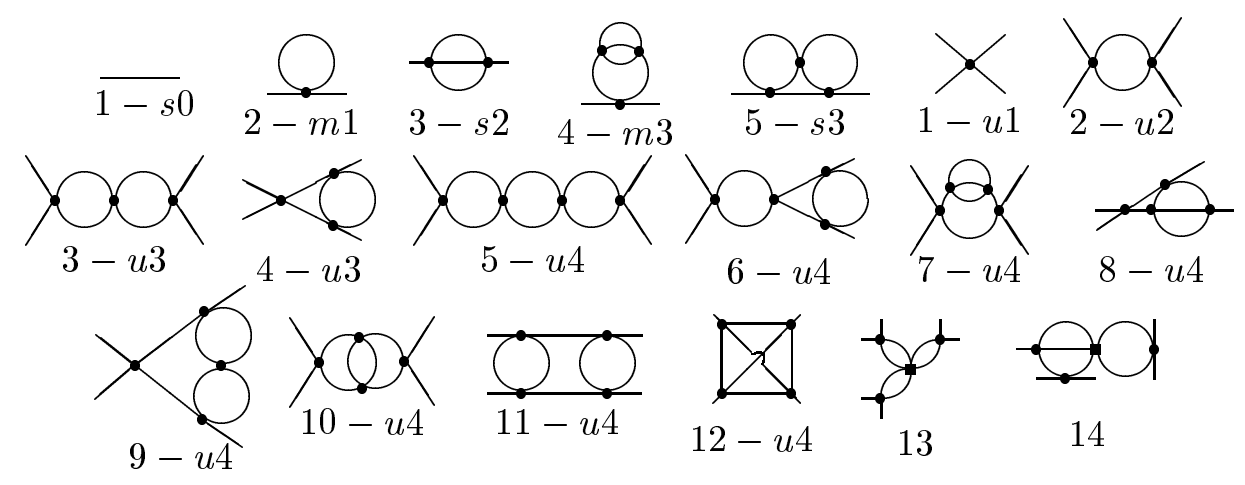

Figure 6. Graphs of functions $\Gamma^{(2)}, \Gamma^{(4)}$ in three-loop approximation. Graphs 13 and 14 represent additional contribution to the function $\Gamma^{(* f)}$. 


\section{B Three Loop Contributions}

In this appendix we have collected the more lenghty expressions for the three loop contributions to RG functions and exponents. The coefficients $b_{v_{12}}^{j}$ for the $\tau$ expansion of the function $\beta_{v_{12}}^{m}$ (4.4) read:

$$
\begin{aligned}
& b_{v_{12}}^{0}=-\frac{20 v_{22}{ }^{3}}{27}-\frac{20 v_{11}{ }^{3}}{27}-\frac{14 v_{12}{ }^{3}}{27}-\frac{26 v_{12}{ }^{2} v_{22}}{27}-\frac{28 v_{12} v_{22}{ }^{2}}{27}- \\
& \frac{28 v_{12} v_{11}^{2}}{27}-\frac{26 v_{12}^{2} v_{11}}{27}-\frac{16 v_{12} v_{2} v_{11}}{27} \\
& b_{v_{12}}^{1}=\frac{70 v_{11}^{3}}{27}+\frac{70 v_{22}{ }^{3}}{27}+\frac{52 v_{12}^{3}}{27}+\frac{32 v_{12} v_{22}^{2}}{9}+\frac{32 v_{12} v_{11}^{2}}{9}+ \\
& \frac{88 v_{12}^{2} v_{22}}{27}+\frac{88 v_{12}^{2} v_{11}}{27}+\frac{60 v_{12} v_{22} v_{11}}{27}, \\
& b_{v_{12}}^{2}=\frac{4 v_{11}^{3}}{9}+\frac{4 v_{22}{ }^{3}}{9}+\frac{v_{22}{ }^{3} d}{18}-\frac{4 v_{12} v_{22}^{2}}{27}-\frac{4 v_{12} v_{11}^{2}}{27}+ \\
& \frac{v_{11}^{3} d}{18}+\frac{v_{12} v_{11}^{2} d}{18}+\frac{v_{12} v_{22}^{2} d}{18}, \\
& b_{v_{12}}^{3}=-\frac{2 v_{11}^{3}}{9}-\frac{2 v_{22}{ }^{3}}{9}-\frac{2 v_{12} v_{11}^{2}}{9}-\frac{2 v_{12} v_{22}^{2}}{9} \text {, } \\
& b_{v_{12}}^{4}=-\frac{16 v_{11}^{3}}{9}-\frac{16 v_{22}{ }^{3}}{9}-\frac{20 v_{12}^{2} v_{11}}{9}-\frac{20 v_{12}{ }^{2} v_{22}}{9}-\frac{16 v_{12} v_{11}^{2}}{9}- \\
& \frac{16 v_{12} v_{22}^{2}}{9}-\frac{8 v_{12}{ }^{3}}{9}-\frac{8 v_{12} v_{22} v_{11}}{9}, \\
& b_{v_{12}}^{5}=-\frac{12 v_{11}^{3}}{27}-\frac{12 v_{22}{ }^{3}}{27}-\frac{2 v_{12}{ }^{2} v_{11}}{3}-\frac{2 v_{12}^{2} v_{22}}{3}-\frac{4 v_{12} v_{11}{ }^{2}}{3}- \\
& \frac{4 v_{12} v_{22}^{2}}{3}-\frac{2 v_{12}^{3}}{3}-\frac{8 v_{12} v_{22} v_{11}}{9}, \\
& b_{v_{12}}^{6}=-\frac{v_{11}{ }^{3}}{3}-\frac{v_{22}{ }^{3}}{3}-\frac{2 v_{12}^{2} v_{11}}{9}-\frac{2 v_{12}^{2} v_{22}}{9}-\frac{2 v_{12}{ }^{3}}{9}-\frac{2 v_{12} v_{22} v_{11}}{9} \text {, } \\
& b_{v_{12}}^{7}=-\frac{4 v_{12}^{2} v_{11}}{9}-\frac{4 v_{12}^{2} v_{22}}{9}-\frac{2 v_{12} v_{11}^{2}}{3}-\frac{2 v_{12} v_{22}{ }^{2}}{3}-\frac{2 v_{12}{ }^{3}}{9}, \\
& b_{v_{12}}^{8}=-\frac{4 v_{11}^{3}}{9}-\frac{4 v_{22}^{3}}{9} \text {. }
\end{aligned}
$$

The coefficients $\eta_{G}^{j}$ introduced by equation (5.11) giving the $\tau$ expansion of the exponent $\eta_{f_{1} f_{2}}^{\mathrm{G}}$ in the Gaussian fixed point $\mathrm{G}$ read:

$$
\begin{aligned}
& \eta_{G}^{0}=-\frac{3 f_{1}{ }^{2} f_{2}{ }^{2}}{8}-\frac{7 f_{1} f_{2}}{8}+\frac{3 f_{2} f_{1}{ }^{2}}{4}+\frac{3 f_{1} f_{2}{ }^{2}}{4}-\frac{f_{2} f_{1}{ }^{3}}{8}-\frac{f_{1} f_{2}{ }^{3}}{8}, \\
& \eta_{G}^{1}=\frac{3 f_{1}{ }^{2} f_{2}{ }^{2}}{2}+\frac{3 f_{1} f_{2}{ }^{3}}{8}+\frac{3 f_{2} f_{1}{ }^{3}}{8}-\frac{23 f_{1} f_{2}{ }^{2}}{8}-\frac{23 f_{2} f_{1}{ }^{2}}{8}+\frac{15 f_{1} f_{2}}{4}, \\
& \eta_{G}^{2}=0, \quad \eta_{G}^{3}=0, \\
& \eta_{G}^{4}=-\frac{3 f_{2} f_{1}^{3}}{8}-\frac{3 f_{1} f_{2}{ }^{3}}{8}+\frac{9 f_{1} f_{2}{ }^{2}}{8}-\frac{3 f_{1}{ }^{2} f_{2}{ }^{2}}{4}+\frac{9 f_{2} f_{1}{ }^{2}}{8}
\end{aligned}
$$




$$
\begin{aligned}
& \eta_{G}^{5}=-\frac{3 f_{1}{f_{2}}^{2}}{8}-\frac{3 f_{1}{ }^{2} f_{2}{ }^{2}}{8}+\frac{15 f_{1} f_{2}}{8}-\frac{3 f_{2} f_{1}{ }^{2}}{8}, \\
& \eta_{G}^{6}=\frac{3 f_{2} f_{1}{ }^{2}}{8}+\frac{3 f_{1} f_{2}{ }^{2}}{8}-\frac{3 f_{1} f_{2}}{8}-\frac{3 f_{1}{ }^{2}{f_{2}}^{2}}{8}, \\
& \eta_{G}^{7}=-\frac{3 f_{2} f_{1}{ }^{2}}{8}+\frac{9 f_{1} f_{2}}{8}-\frac{3 f_{1} f_{2}{ }^{2}}{8}, \quad \eta_{G}^{8}=0 .
\end{aligned}
$$

The coefficients $\eta_{U}^{j}$ introduced by equation (5.12) giving the $\tau$ expansion of the exponent $\eta_{f_{1} f_{2}}^{U}$ in the unsymmetric fixed point $\mathrm{U}$ read:

$$
\begin{aligned}
& \eta_{U}^{0}=-\frac{81 f_{1}{ }^{2} f_{2}{ }^{2}}{256}-\frac{27 f_{1} f_{2}{ }^{3}}{512}-\frac{153 f_{2} f_{1}{ }^{3}}{512}-\frac{35 f_{1}{ }^{2}}{64}+\frac{41 f_{1}}{128}-\frac{41 f_{1} f_{2}}{32}+ \\
& \frac{285 f_{1} f_{2}{ }^{2}}{512}+\frac{17 f_{1}{ }^{3}}{64}+\frac{673 f_{2} f_{1}{ }^{2}}{512}-\frac{5 f_{1}{ }^{4}}{128}, \\
& \eta_{U}^{1}=\frac{297 f_{1}{ }^{2} f_{2}{ }^{2}}{256}+\frac{81 f_{1} f_{2}{ }^{3}}{512}+\frac{549 f_{2} f_{1}{ }^{3}}{512}+\frac{1427 f_{1} f_{2}}{256}-\frac{2671 f_{2} f_{1}{ }^{2}}{512}- \\
& \frac{1077 f_{1} f_{2}{ }^{2}}{512}-\frac{67 f_{1}{ }^{3}}{64}-\frac{185 f_{1}}{128}+\frac{301 f_{1}{ }^{2}}{128}+\frac{9 f_{1}{ }^{4}}{64}, \\
& \eta_{U}^{2}=-\frac{11 f_{2} f_{1}{ }^{2}}{128}-\frac{3 f_{1} f_{2}{ }^{2}}{128}+\frac{23 f_{1} f_{2}}{128}-\frac{f_{1}}{8}+\frac{5 f_{1}{ }^{2}}{32}-\frac{f_{1}{ }^{3}}{32}, \quad \eta_{U}^{3}=0 \\
& \eta_{1}^{4}=-\frac{369 f_{2} f_{1}{ }^{3}}{512}-\frac{81 f_{1} f_{2}{ }^{3}}{512}+\frac{405 f_{1} f_{2}{ }^{2}}{512}-\frac{189 f_{1}{ }^{2} f_{2}{ }^{2}}{256}+\frac{819 f_{2} f_{1}{ }^{2}}{512}+ \\
& \frac{51 f_{1} f_{2}}{256}+\frac{21 f_{1}{ }^{3}}{64}-\frac{3 f_{1}{ }^{4}}{32}-\frac{15 f_{1}}{64}, \\
& \eta_{U}^{5}=-\frac{27 f_{1}{ }^{2} f_{2}{ }^{2}}{128}+\frac{405 f_{1} f_{2}}{256}-\frac{27 f_{1} f_{2}{ }^{2}}{128}-\frac{63 f_{2} f_{1}{ }^{2}}{256}-\frac{45 f_{2} f_{1}{ }^{3}}{256}- \\
& \frac{123 f_{1}}{256}-\frac{3 f_{1}{ }^{3}}{64}+\frac{141 f_{1}{ }^{2}}{256}-\frac{3 f_{1}{ }^{4}}{128}, \\
& \eta_{U}^{6}=\frac{189 f_{2} f_{1}{ }^{2}}{256}+\frac{27 f_{1} f_{2}{ }^{2}}{128}-\frac{147 f_{1} f_{2}}{256}-\frac{27 f_{1}{ }^{2} f_{2}{ }^{2}}{128}-\frac{45 f_{2} f_{1}{ }^{3}}{256}+ \\
& \frac{33 f_{1}}{256}-\frac{63 f_{1}{ }^{2}}{256}+\frac{9 f_{1}{ }^{3}}{64}-\frac{3 f_{1}{ }^{4}}{128}, \\
& \eta_{U}^{7}=-\frac{297 f_{2} f_{1}{ }^{2}}{512}+\frac{663 f_{1} f_{2}}{512}-\frac{135 f_{1} f_{2}{ }^{2}}{512}-\frac{89 f_{1}}{256}+\frac{117 f_{1}{ }^{2}}{256}-\frac{7 f_{1}{ }^{3}}{64} \\
& \eta_{U}^{8}=\frac{3 f_{1} f_{2}}{64}+\frac{3 f_{1}{ }^{2}}{64}-\frac{3 f_{1}}{64}, \\
& \eta_{U}^{12}=-\frac{7 f_{1}{ }^{2}}{16}+\frac{f_{1}{ }^{3}}{16}+\frac{3 f_{1}}{8}+\frac{11 f_{2} f_{1}{ }^{2}}{64}+\frac{3 f_{1} f_{2}{ }^{2}}{64}-\frac{31 f_{1} f_{2}}{64} . \\
& \hline{ }^{2}
\end{aligned}
$$

The coefficients $\eta_{M A W}^{j}$ introduced by equation (5.13) giving the $\tau$ expansion of the exponent $\eta_{f}^{M A W}$ for mutually avoiding walks read:

$$
\eta_{M A W}^{0}=\frac{13 f}{8}-\frac{5 f^{4}}{16}-\frac{49 f^{2}}{16}+\frac{7 f^{3}}{4},
$$




$$
\begin{aligned}
& \eta_{M A W}^{1}=\frac{9 f^{4}}{8}+\frac{97 f^{2}}{8}-\frac{53 f^{3}}{8}-\frac{53 f}{8}, \\
& \eta_{M A W}^{2}=0, \quad \eta_{M A W}^{3}=0, \\
& \eta_{M A W}^{4}=-\frac{9 f^{2}}{2}+\frac{27 f^{3}}{8}+\frac{15 f}{8}-\frac{3 f^{4}}{4}, \\
& \eta_{M A W}^{5}=-\frac{3 f}{4}-\frac{3 f^{4}}{16}+\frac{3 f^{3}}{8}+\frac{9 f^{2}}{16}, \\
& \eta_{M A W}^{6}=\frac{9 f}{8}-\frac{3 f^{4}}{16}-\frac{33 f^{2}}{16}+\frac{9 f^{3}}{8}, \\
& \eta_{M A W}^{7}=-\frac{19 f}{16}+\frac{27 f^{2}}{16}-\frac{f^{3}}{2}, \quad \eta_{M A W}^{8}=0 .
\end{aligned}
$$

\section{References}

[1] J. des Cloizeaux and G. Jannink. Polymers in Solution. Clarendon Press, Oxford, 1990.

[2] B. Duplantier. J. Stat. Phys., 54:581, 1989.

[3] P. G. de Gennes. Phys. Lett. A, 38:339, 1972.

[4] P.-G. de Gennes. Scaling Concepts in Polymer Physics. Cornell University Press, Ithaca and London, 1979.

[5] L. Leibler J.-F. Joanny and R. Ball. J. Chem. Phys., 81:4640, 1984.

[6] J.F. Douglas and K.F. Freed. J. Chem. Phys., 86:4280, 1987.

[7] L. Schäfer and Ch. Kappeler. J. Phys. (Paris), 46:1853, 1985.

[8] L. Schäfer and C. Kappeler. Colloid Polym. Sci., 268:995, 1990.

[9] J. Roovers J.F. Douglas and K.F. Freed. Macromolecules, 23:4168, 1990.

[10] U. Lehr L. Schäfer and C. Kappeler. J. Phys. (Paris) I, 1:211, 1991.

[11] F. S. Bates and G. H. Fredrickson. Annu. Rev. Phys. Chem., 41:525, 1990.

[12] B. Duplantier. Phys. Rev. Lett., 57:941, 1986.

[13] U. Lehr L. Schäfer, C. von Ferber and B. Duplantier. Nucl. Phys. B, 374:473, 1992.

[14] D. J. Wallace and R. K. P. Zia. J. Phys. C, 8:839, 1975.

[15] A. Myake and K. F. Freed. Macromolecules, 16:1228, 1983.

[16] K. Ohno and K. Binder. J. Phys. (Paris), 49:1329, 1988.

[17] H. Saleur. J. Phys. A, 19:L807, 1986.

[18] A. J. Barrett and D.L. Tremain. Macromolecules, 20:1687, 1987.

[19] J. Batoulis and K. Kremer. Macromolecules, 22:4277, 1989.

[20] P. Grassberger. Nonuniform star polymers in 2 dimensions. preprint, 1994.

[21] M. E. Cates and T. A. Witten. Phys. Rev. A, 35:1809, 1987.

[22] C. von Ferber and Y. Holovatch. Field theoretical approach to higher order calculations of a multifractal spectrum. preprint, 1996.

[23] J. C. Le Guillou E. Brezin and J. Zinn-Justin. In C.Domb and M.S.Green, editors, Phase transitions and critical phenomena, volume 6, pages 125-247. Academic Press, New York, 1976.

[24] G. Parisi. J. Stat. Phys., 23:49, 1980.

[25] C. von Ferber and Y. Holovatch. Copolymer Networks: Multifractal dimension spectra in polymer field theory preprint, 1996 .

[26] To give a meaning to the exponential of $\delta$-functions an appropriate short distance cutoff is introduced in the integrations.

[27] S. F. Edwards. Proc. Phys. Soc., 85:613, 1965.

[28] S. F. Edwards. Proc. Phys. Soc., 88:265, 1966.

[29] Duplantier B. Commun. Math. Phys. 117:279, 1988; Duplantier B. and Kwon K.-H. Phys. Rev. Lett. 61:2514, 1988. 
[30] N. N. Bogoliubov and D. V. Shirkov. Introduction to the theory of quantized fields. Wiley \& Sons, New York, 1959.

[31] D. J. Amit. Field Theory, the Renormalization Group, and Critical Phenomena. World Scientific, Singapore, 2.ed.1984.

[32] K.G. Wilson and M.E. Fisher. Phys. Rev. Lett., 28:240, 1972.

[33] E. Brézin, J.C. Le Guillou, J. Zinn-Justin and B.G. Nickel. Phys. Lett. A, $44: 227,1973$

[34] A.A. Vladimirov, D.I. Kazakov and O.V. Tarasov. Sov. Phys. JETP, 77:1035, 1979.

[35] S.G. Gorishny, S.A. Larin and F.V. Tkachov. Phys. Lett. A, 101:120, 1984.

[36] B.G. Nickel G.A. Baker, Jr. and D.I. Meiron. Phys. Rev. B, 17:1365, 1978.

[37] J. Zinn-Justin J. C. Le Guillou. Phys. Rev. B, 21:3976, 1980.

[38] C. Bagnuls and C. Bervillier. Phys. Rev. B, 32:7209, 1985.

[39] C. Bagnuls and C. Bervillier. Phys. Rev. B, 35:3585, 1987.

[40] See formula (9.8) of reference [23] for instance.

[41] Note that expression (4.3) gives the dimensional dependence of the $\beta$-function of $O(m)$-symmetric $\phi^{4}$ model at $m=0$ in three -loop order. In order to compare this with expression for the $\bar{\beta}$-function of three-dimensional theory (e.g. formula (2.5)) of [36]) one should substitute loop integrals $i_{1}-i_{8}$ entering (4.3) by their values at $d=3$ (see $[42,43]$ and appendix $\mathrm{A}$ of this article).

[42] D.I. Meiron B.G. Nickel and Jr. G.A. Baker. Compilation of 2-pt. and 4-pt. graphs for continuous spin models. University of Guelf Report, 1977.

[43] Yu. Holovatch and T. Krokhmalskii. Journ.Math.Phys., 35:3866, 1994.

[44] The method of numerically solving the fixed point equation for the resummed $\beta$-function in $O(m)$-symmetric $\phi^{4}$ theory with one coupling in the massive scheme was initiated in [36] and then intensively exploited in theories with several couplings, describing diluted Ising and cubic anisotropy models (G. Jug, Phys. Rev. B 27:609,1983; I.O. Mayer, A.I. Sokolov and B.N. Shalaev, Ferroelectrics 95:93, 1989; I.O. Mayer, J. Phys. A 22:2815, 1989; Yu. Holovatch and M. Shpot, J. Stat. Phys., 66:867, 1992), mn-model (N.A. Shpot, Phys. Lett. A,142:474, 1989), anisotropic superconducting and magnetic systems with vector order parameters (S.A. Antonenko and A.I. Sokolov, Phys.Rev.B 49:15901, 1994: S.A. Antonenko, PhD Thesis, A.F. Ioffe Physico-Technical Institute, St. Petersburg, 1995.)

[45] First introduced by B.G. Nickel: see ref.19 in [37].

[46] J. C. Le Guillou E. Brézin and J. Zinn-Justin. Phys. Rev. D, 15:1544, 1977.

[47] L.N. Lipatov. Sov. Phys. JETP., 45:216, 1977.

[48] E. Brezin and G. Parisi. J. Stat. Phys., 19:269, 1978.

[49] G.H. Hardy. Divergent Series. Oxford University, Oxford, 1948.

[50] J. Zinn-Justin. Euclidean Field Theory and Critical Phenomena. Oxford University Press, New York, 1989.

[51] C. Itzykson and J. B. Zuber. Quantum Field Theory. McGraw-Hill, New York, 1980

[52] P. Graves-Morris G.A. Baker, Jr. Padé Approximants. Part I: Basic Theory. Part II: Extensions and Applications. Addison-Wesley Publishing Co, Reading Massachusetts, 1981.

[53] The value for the two-loop approximation used here was obtained in [54]. Due to different normalizations $u^{*}\left(\right.$ Ref.[54]) $=\frac{4}{3} v_{a a}^{*}$ (here) $=1.5809$. In three loop approximation the corresponding value equals $u^{*}=1.4393$ [54], in six loops one has $u^{*}=1.421 \pm 0.004[36]$.

[54] Yu. Holovatch Int.Journ.Mod.Phys. A, 8:5329, 1993.

[55] In fact the system of equations for the fixed point is solved at fixed order of perturbation theory (in our example we use a two-loop approximation). With increasing order the difference between the results will decrease.

[56] C. von Ferber and Y. Holovatch. Theor. Mat. Phys. (Moscow), to appear, 1996.

[57] V. G. Kac. In Lecture Notes in Physics, volume 94, page 441. Springer Verlag, 1979. 
[58] A.M. Polyakov A.A. Belavin and A.B. Zamolodchikov. Nucl. Phys. B, 241:333, 1984.

[59] Z. Qiu D. Friedan and S. Shenker. Phys. Rev. Lett., 52:1575, 1984.

[60] L. P. Kadanoff I. Procaccia T. C. Halsey, M. H. Jensen and B.I. Shraiman. Phys. Rev. A, 33:1141, 1986.

[61] A. W. W. Ludwig and B. Duplantier. Phys. Rev. Lett., 66:247, 1991.

\title{
СТАТИСТИКА БАГАТОКОМПОНЕНТНИХ ПОЛІМЕРНИХ ЗІРОК
}

\author{
К. фон Фербер, Ю.Головач
}

\begin{abstract}
Ми досліджуємо полімерну сітку, утворену хемічно різними полімерами. Для опису масштабних властивостей зіркоподібних вершин, що її утворюють, ми вводимо новий набір критичних показників. У випадку сітки полімерів двох сортів ми називаємо ці показники кополімерними зірковими показниками. На основі опису нашої теорії за допомогою лагранжевої теорії поля ми обчислюємо у третьому порядку теорії збурень ці показники а також показники, що описують масштабні властивості зірки утвореної блуканнями з взаємним униканням. У випадку однорідних зірок ми отримуємо раніше відомі результати. У деяких частинних випадках наші результати також узгоджуються 3 попередньо відомими результати отриманими у другому порядку $\epsilon$-розкладу. Виявлено стабільність і узгодженість результатів при $d=2$ і $d=3$ із сподіваним зростанням відхилень для великої кількості променів однієї зірки. Такі ж методи, застосовані раніше до задачі про однорідні зіркові показники, привели до результатів, що добре узгоджуються із обчисленнями, проведеними методом Монте Карло. Ми сподіваємося, що наші обчислення також стимулюватимуть дослідження кополімерних зірок за допомогою методу Монте Карло. Пересумовані значення показників для зірок утворених блуканнями із взаємним униканням добре узгоджуються із точними результатами запропонованими раніше для $d=2$. Наші дослідження випадку $d=2$ можуть сприяти дослідженню проблеми формулювання нашої теорії на мові двовимірної конформної теорії поля. Досліджуючи властивості опуклості спектру кополімерних зіркових показників ми показуємо, що вони є добрими кандидатами для застосування у теорії мультифрактального спектру, утвореного гармонійною дифузією біля фрактального абсорбеpa.
\end{abstract}

